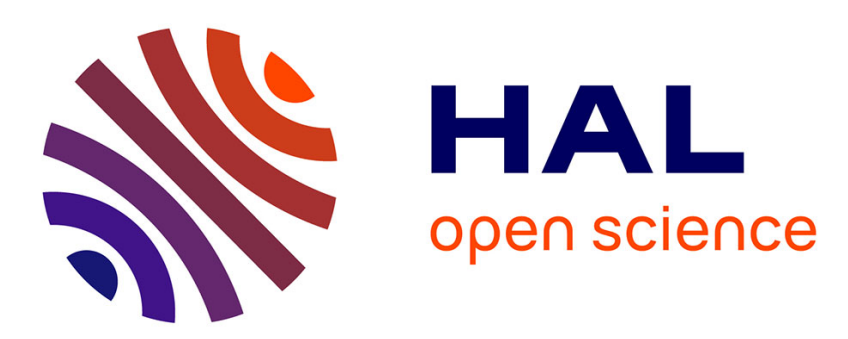

\title{
A nonlinear Lagrangian particle model for grains assemblies including grain relative rotations
}

Emilio Turco, Francesco Dell'Isola, Anil Misra

\section{To cite this version:}

Emilio Turco, Francesco Dell'Isola, Anil Misra. A nonlinear Lagrangian particle model for grains assemblies including grain relative rotations. International Journal for Numerical and Analytical Methods in Geomechanics, 2019, Special Issue: Special Issue on Particle Methods in Geomechanics, 43 (5), pp.1051-1079. hal-02269692

\section{HAL Id: hal-02269692 \\ https://hal.science/hal-02269692}

Submitted on 23 Aug 2019

HAL is a multi-disciplinary open access archive for the deposit and dissemination of scientific research documents, whether they are published or not. The documents may come from teaching and research institutions in France or abroad, or from public or private research centers.
L'archive ouverte pluridisciplinaire HAL, est destinée au dépôt et à la diffusion de documents scientifiques de niveau recherche, publiés ou non, émanant des établissements d'enseignement et de recherche français ou étrangers, des laboratoires publics ou privés. 


\title{
A nonlinear Lagrangian particle model for grains assemblies including grain relative rotations
}

\author{
Emilio Turco' ${ }^{1}$ | Francesco dell'Isola ${ }^{2} \mid$ Anil Misra $^{3}$
}

${ }^{1}$ Department of Architecture, Design and Urban planning (DADU), University of Sassari and M\&MOCS International Research Center, Alghero, Italy

${ }^{2}$ Department of Structural and Geotechnical Engineering, University of Roma "La Sapienza" and M\&MOCS International Research Center, Rome, Italy ${ }^{3}$ Civil, Environmental and Architectural Engineering (CEAE), University of Kansas School of the Arts, Lawrence, Kansas,

\section{Correspondence}

Emilio Turco, Department of

Architecture, Design and Urban planning (DADU), University of Sassari and

M\&MOCS International Research Center, Alghero, Italy.

Email: emilio.turco@uniss.it

\begin{abstract}
Summary
We formulate a discrete Lagrangian model for a set of interacting grains, which is purely elastic. The considered degrees of freedom for each grain include placement of barycenter and rotation. Further, we limit the study to the case of planar systems. A representative grain radius is introduced to express the deformation energy to be associated to relative displacements and rotations of interacting grains. We distinguish inter-grains elongation/compression energy from inter-grains shear and rotations energies, and we consider an exact finite kinematics in which grain rotations are independent of grain displacements. The equilibrium configurations of the grain assembly are calculated by minimization of deformation energy for selected imposed displacements and rotations at the boundaries. Behaviours of grain assemblies arranged in regular patterns, without and with defects, and similar mechanical properties are simulated. The values of shear, rotation, and compression elastic moduli are varied to investigate the shapes and thicknesses of the layers where deformation energy, relative displacement, and rotations are concentrated. It is found that these concentration bands are close to the boundaries and in correspondence of grain voids. The obtained results question the possibility of introducing a first gradient continuum models for granular media and justify the development of both numerical and theoretical methods for including frictional, plasticity, and damage phenomena in the proposed model.
\end{abstract}

\section{KEYWORDS}

discrete models, granular media, mechanics of materials, nonlinear analysis

\section{1 | INTRODUCTION}

In a large number of engineering applications, it is necessary to describe the behaviour of granular media and to understand the intrinsic mechanical phenomena involved in the deformation of grains assembly. The study of the mechanics of granular media is obviously relevant in geotechnics, for instance to design and control earth structures, tunnels, and ground improvement. Mechanics of granular materials are also relevant to the control of powder processing procedures, ${ }^{1}$ to guide the production of pharmaceutical tablet, ${ }^{2}$ or to understand performance of granular membranes frequently used in civil engineering or to conceive bio-membranes. ${ }^{3}$ The intrinsic complexity of considered systems and the related deformation phenomena require a fresh revisitation of the used methods and, in our opinion, a revival of the basic Lagrangian methods. 
We explicitly and immediately remark that we limit our attention to discrete (ie, finite dimensional) models in which grains (i) have a configuration characterised by their barycenter placement and their rotation and (ii) are interacting pairwise with elastic energy depending upon their relative rotation, displacement, and consequent relative shear. We believe that, even if dissipative and, in general, nonconservative phenomena play a relevant role in the mechanics of grain assemblies, a clearly specified Lagrangian model is an important starting point in the proposed investigation. Indeed, conservative deformation phenomena always occur and are of relevance, while the incremental balance of force approach, sometimes used in the literature, see, eg, Cundall and Strack ${ }^{4}$ does not clearly distinguish between conservative and dissipative interaction forces.

Indeed, one of the most influential paper in the field has been Cundall and Strack. ${ }^{4}$ In it, Cundall and Strack formulated what is now known as the discrete element model (DEM) in which the behaviour of assembly of grains is reduced to the mechanical interaction of an assembly of discs, subject to mutual forces. In mentioned paper, there is no reference to conservation of elastic energy, and the integration scheme used is based on an incremental sequential determination of the solution of the Newton force balance, calculated grain by grain, time step after time step. This concept is being still used, eg, in Kuhn ${ }^{5}$ and Radja and Dubois ${ }^{6}$; however, occasionally, a variational point of view is accepted in the literature (see Holtzman et $\mathrm{al}^{7}$ ). We also note that in the classical DEM algorithms, the grain interactions are treated from the viewpoint of Hertz-Cattaneo-Mindlin contact analysis. In geomaterials, however, grain-interactions are often mediated via certain cementation, multi-asperity contacts, nano/microscopic bridges/welds, or long-range/noncontact mechanisms. The treatment of such grain-interactions as nearly point contacts, following the Hertz-Cattaneo-Mindlin paradigm, may not completely capture all the relevant deformation mechanisms.

Rather inexplicably in the literature, one finds very few experiments trying to point out and highlight the most fundamental features of the mechanics of grains assemblies: Among these, we cite Misra and Jiang ${ }^{8}$ and Richefeu et al. ${ }^{9}$ Experiments that measure grain-scale behaviour are few and are limited. Systematic studies of the measurements of grain trajectories (including grain translation and spin) in well-defined granular system are rare, see for example trajectory measurements in certain assemblies of regularly arranged grains, see Misra and Poorsolhjouy. ${ }^{10}$ Notably, studies that combine trajectory measurements with forces, such as "force chains," are particularly rare. The limited experiments, and also the available numerical simulations based on every variant of DEM, indicate that it is not so easy to formulate a continuum macroscopic model that can capture the behaviour of granular media and for sure a standard Cauchy first gradient continuum is not suitable to capture the main mechanical phenomena involved in the deformation of a large class of granular media. While there is an abundance of elasto-plastic models, continuum models that link to the grain-scale mechanisms remain work-in-progress as noted in the discussion related to recent papers authored by Misra and his colleagues, see Misra et al. ${ }^{11}$ In this regard, we also point to the recently proposed micromorphic continuum models ${ }^{12}$ which follow, in the spirit of Piola ansatz, ${ }^{13}$ from an identification of the grain-scale motions in terms of the small deformation continuum kinematic measures, and the volume average of grain-pair interaction energies with macro-deformation energy density, and demonstrate clearly the necessity for enhanced continuum models for granular materials. ${ }^{14,15}$

In a similar context, recently, the relationship between continuum homogenised models and their discrete microscopic counterparts has been reexamined and revisited with a view towards the numerical applications and the need of getting effective and efficient numerical codes: see, eg, Turco ${ }^{16}$ for Lagrangian discrete models for spatial beams in large deformations, ${ }^{17}$ dealing with 2D networks of beams, and Turco and Caracciolo ${ }^{18}$ which considers a concentrated elasticity model for plates. It is remarkable that there are many similarities between these models for structural elements or complex microstructures and approaches that can be introduced to model grains packages. These common modeling idea consists in describing structural elements or complex structures as an assembly of rigid bodies (with the associated reference system) and elastic connections, which may undergo large deformations. Among the most famous scholar who contributed to advancing this idea was Hencky, see Hencky, ${ }^{19}$ who exploited such models for the evaluation of buckling loads of two-dimensional beams.*

In the present work, we develop a novel Lagrangian discrete model for planar assemblies of grains, which is able to investigate the evolution of a quasi-static deformation process, see Turco. ${ }^{20}$ In this first effort, we do not dismiss completely the the method traced by Cundall and Strack. We postulate, however, an expression of deformation energy, which depends on the relative displacement and relative rotation of two close interacting grains, and in particular, we clearly define the concept of shear deformation relative to the relative grain roto-translation, which we could not easily find in the literature. We assume that each grain is characterised by a typical radius in terms of which we describe the relative inter-granular deformation energy: This radius is needed to find the fundamental state relative placement of every

* It has to be noted that Gabrio Piola ${ }^{13}$ did justify the Euler-Bernoulli choice of deformation energy for an Elastica with a similar argument. 


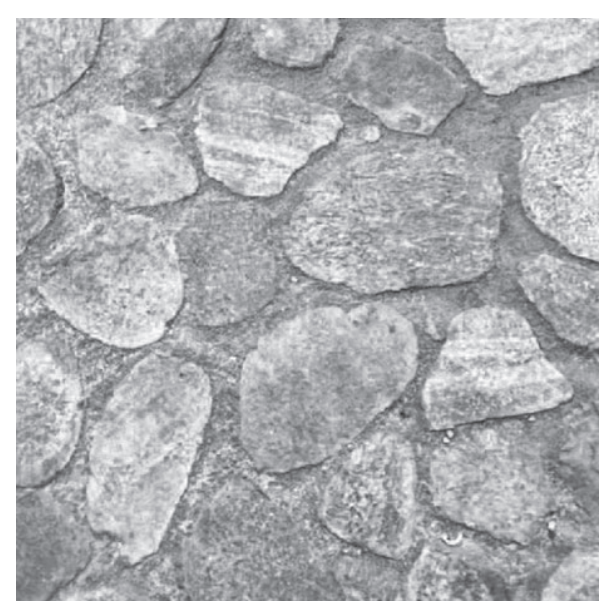

(A)

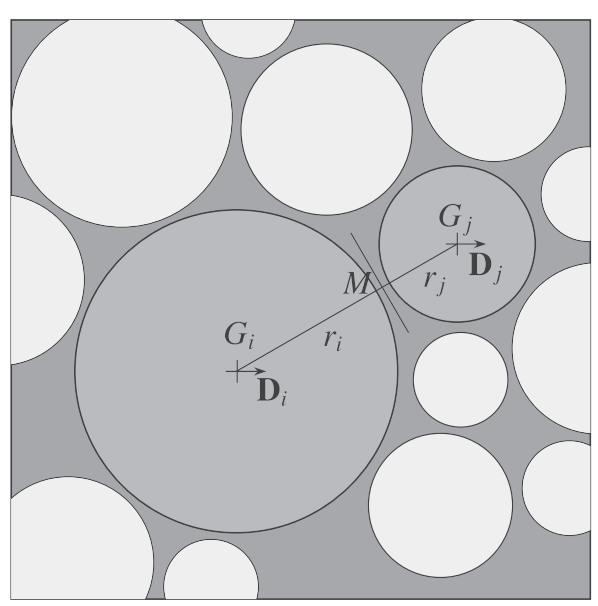

(B)

FIGURE 1 A, Buried grains in an elastic matrix and B, its schematic representation

interacting pair of grains. We assume that the elastic deformation occurring in the elastic grains is effectively described, wherever it may occur or be localised, in terms of introduced relative rotation, shear, and elongation. This generalises the Hertzian approach to grain interactions in which one assumes that the elastic interaction is localised at grain interfaces. We believe that the interesting considerations developed in the previous studies ${ }^{21-23}$ can be completed with those presented here. In this introduction, we have presented a subjective selection of those which we consider the main references about the mechanics of granular materials from a rather vast literature base. Furthermore, the main characteristics of the presented model are briefly discussed trying to depict its peculiarities.

We neglect every dynamical effects, and we assume therefore that the inter-granular mechanical interaction can be given by means of a variational formulation, based on a postulated form of the local strain energy: see Section 2 . We investigate the response to quasi-static transformations. These responses are of practical interest and are also more easily computable using a step-wise computation method, which manages to handle effectively also large deformation processes, even if quasistatic simulations can require the solution of remarkable numerical challenges as they may present instabilities and bifurcation phenomena. We have chosen to use a stepwise procedure based on Newton's method as predictor and Riks' arc-length idea as corrector. In this way, we could effectively and systematically reconstruct completely the equilibrium path when a specific parameter is increased. In this way, we overcome the critical points which may be encountered in the evaluation process, see Section 3. We present the results of performed numerical simulations, and we conclude that they indicate that the model is promising, although it needs to be improved and generalised, see Section 4 . We also describe shortly some of the future challenges, which we believe deserve particular attention, see Section 5.

\section{2 | INTERACTION BETWEEN GRAINS}

We consider a package of grains buried in an elastic matrix such as that shown in Figure 1A. We can represent schematically the package as reported in Figure 1B. Here, the grains $i$ and $j$ are sketched using a circular shape only for the sake of simplified visualization. Their barycentres (in the reference configuration) and radii are $G_{i}, r_{i}, G_{j}$, and $r_{j}$, respectively. Furthermore, for describing the grains rotations, we consider, see again Figure 1, two unit vectors $\mathbf{D}_{i}$ and $\mathbf{D}_{j}$. In order to limit the data necessary to completely describe the considered packages of grains, we choose $\mathbf{D}_{i}=\mathbf{D}_{j}=\mathbf{D}_{1}$ (being $\mathbf{D}_{1}$ the unit vector characteristic of 1-direction, in this case, the horizontal direction) but this hypothesis can be easily removed.

We can describe the motion of the considered pair of grains by means of the following kinematic parameters: the current positions of the barycentres $g_{i}$ and $g_{j}$ and the rotations of each one grain by considering the unit vectors $\mathbf{d}_{i}$ and $\mathbf{d}_{j}$ or, alternatively, by the angles $\alpha_{i}$ and $\alpha_{j}$. Figure 2 reports reference and current configuration of the pair of grains. 


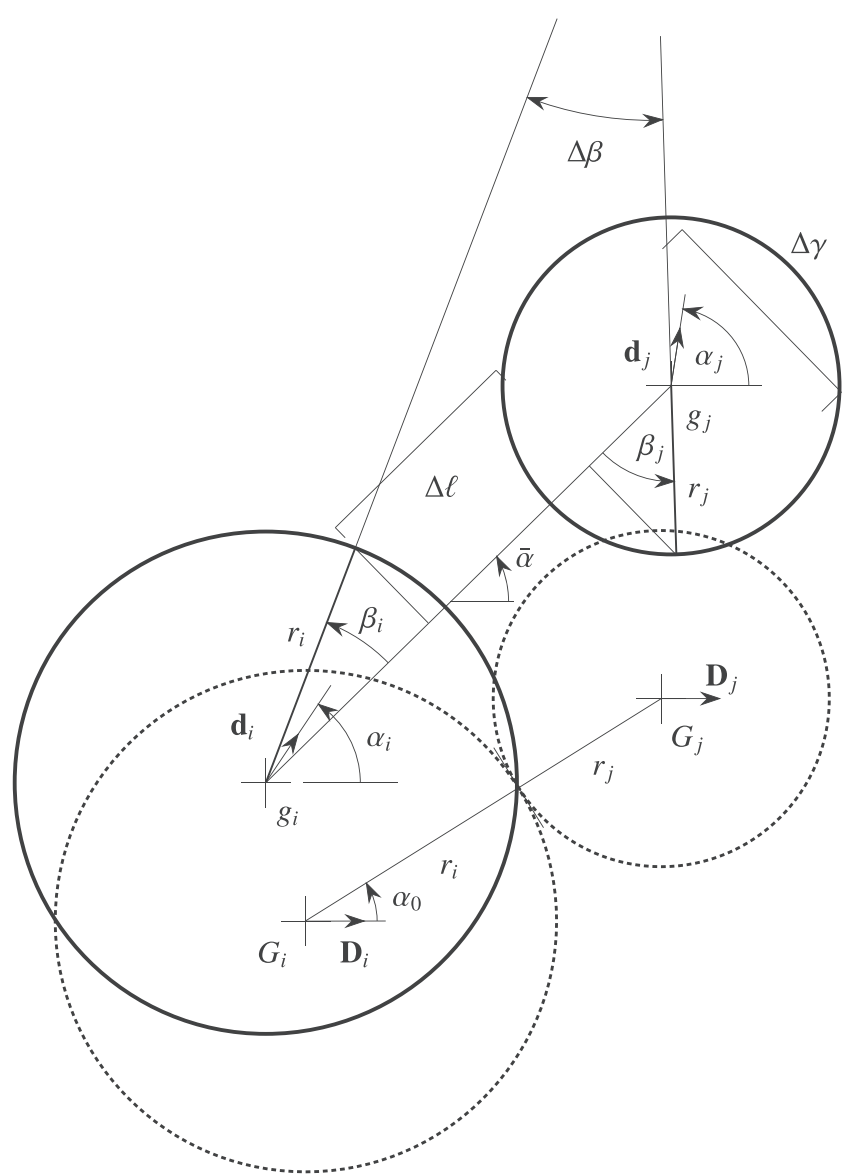

FIGURE 2 Reference (dashed line) and current (continuous line) configuration of a pair of grains: strain measures $\Delta \ell, \Delta \beta$, and $\Delta \gamma$

Limiting ourselves to elastic interactions, the strain energy $E_{i j}$ associated to the pair of grains $i$ and $j$ reported in Figure 2 can be postulated as the simple diagonal quadratic form ${ }^{\dagger}$ :

$$
E_{i j}=\frac{1}{2}\left(a \Delta \ell^{2}+b \Delta \beta^{2}+c \Delta \gamma^{2}\right),
$$

having introduced the constitutive parameters $a, b$, and $c^{\ddagger}$ related to strain measures $\Delta \ell$, stretching, $\Delta \beta$, bending, and $\Delta \gamma$ shearing, respectively, see again Figure 2. We remark here that in large deformations, dissipation could be important as would be tension-compression asymmetry to prevent certain grain inter-penetrations. These aspects will be considered in the future.

The strain measures are worth ${ }^{\S}$ :

$$
\begin{gathered}
\Delta \ell=\left\|g_{j}-g_{i}\right\|+r_{i}\left(1-\cos \beta_{i}\right)+r_{j}\left(1-\cos \beta_{j}\right)-\left(r_{i}+r_{j}\right), \\
\Delta \beta=\beta_{j}-\beta_{i}, \\
\Delta \gamma=r_{i} \sin \beta_{i}+r_{j} \sin \beta_{j},
\end{gathered}
$$

where, beyond the geometric parameters already defined, we used the relationships

$$
\begin{aligned}
& g_{k}=G_{k}+\mathbf{v}_{k}, \\
& \beta_{k}=\alpha_{k}+\alpha_{0}-\bar{\alpha},
\end{aligned}
$$

\footnotetext{
${ }^{\dagger}$ An immediate generalisation could be obtained introducing a nondiagonal quadratic form taking into account, in this case, the interactions among the considered stretching, bending, and shearing energies.

¥They should be determined by means of some suitably chosen experimental tests.

${ }^{\S}$ The Authors are aware that Equation 2 could be simplified. However, they prefer to use this form slightly redundant in order to make the formula closer to kinematic parameters shown in Figure 2 and therefore more readable.
} 


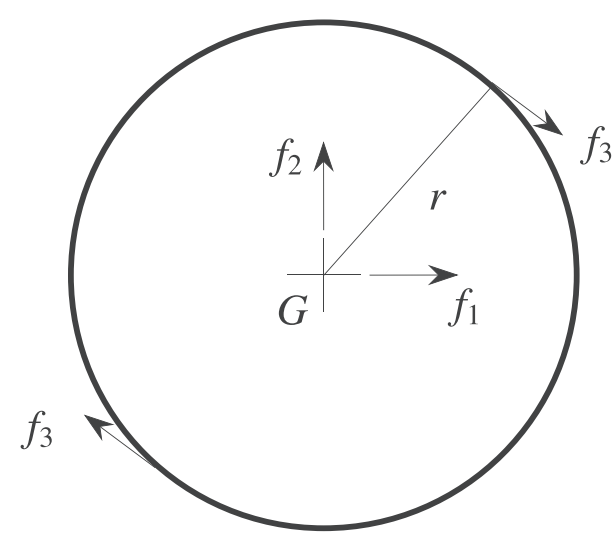

FIGURE 3 Forces acting on a single grain

with $k=i$ or $j$ and $\mathbf{v}_{k}$ collects the two Lagrangian parameters, which express the displacement of the grain $k$. We notice that in Equation 5, the auxiliary angles $\alpha_{0}$ and $\bar{\alpha}$, or their sinuses and cosines, can be evaluated from the knowledge of the geometry and the current position of the grains barycentres, respectively.

We highlight the strong geometrical nonlinearities of the assumed strain energy where, for example, the second and third terms of Equation 2 represent the elongation due to the rotations of the grains. In addition, we also pick out the unusual bending energy term which in the geotechnicians' language is frequently named rocking effect.

It is useful to remark that each grain of the assembly is subjected to a force and a couple usually named structural reaction, or reaction in brief, in the jargon of finite element. In Figure 3, this reaction is represented by the horizontal $f_{1}$ and the vertical $f_{2}$ forces plus a couple force $f_{3}$ whose arm is the diameter of the grain.

\section{3 | HOW TO RECOVER THE EQUILIBRIUM PATH}

The completely discrete model described in the previous section was the base to build an in-house built MatLab code able to get back the whole equilibrium path, ie, a series of pairs, which defines the external loads, forces, or given displacements, and the Lagrangian parameters used to describe the system. The equilibrium path is reconstructed in the framework of a stepwise strategy, in other words, the basic problem is finding an unknown equilibrium point on the basis of the knowledge of the previous one.

If we collect the Lagrangian parameters, which describe the current configuration, eg, the displacement from the reference configuration), in the vector $\mathbf{q}$ then the total strain energy, using Equation 1, becomes $E=E(\mathbf{q})$ and can be obtained just summing the strain energy associated with each pair of interacting grains.

For cases without applied forces, as considered in the numerical simulation presented below, the external load derive from the given displacements. Therefore, the nonlinear system of equilibrium equations, obtained by imposing the stationarity of the global strain energy, reads

$$
\mathbf{s}(\mathbf{q})=\mathbf{0},
$$

being $\mathbf{s}(\mathbf{q})$ the gradient of the global strain energy, generally named reaction, obtained by assembling the elementary contributions deriving from the interaction of each pair of grains.

In the case of given displacements, the Lagrangian parameters used to describe the current configuration, grouped in q, can be split in two parts:

$$
\mathbf{q}=\left[\begin{array}{c}
\mathbf{u} \\
\lambda \overline{\mathbf{u}}
\end{array}\right]
$$

where the vector $\mathbf{u}$ is the collection of the free Lagrangian parameters, and $\lambda \overline{\mathbf{u}}$ of those prescribed using the nondimensional displacement parameter $\lambda$ and the prescribed vector $\overline{\mathbf{u}}$. In other words, the given displacements are all ruled by the nondimensional parameter $\lambda$. We remark that in the framework of an arc-length strategy, it is not possible to limit in advance $\lambda$ as can be done in the framework of a Newton strategy (in this case, $0<\lambda<\lambda_{\max }$, where $\lambda_{\max }$ is fixed in advance). Conversely, in an arc-length strategy only the total length, or, equivalently the number of steps to perform, of the equilibrium path, which we want to reconstruct can be fixed in advance. 
As a consequence, the equilibrium path is a set of pairs $\left(\lambda_{i}, \mathbf{u}_{i}\right)$ close enough to accurately describe the equilibrium path.

The solution of the nonlinear system of equilibrium Equation 6 can be obtained, in the framework of a stepwise strategy, for example by using the Newton's scheme. If the pair $\left(\lambda_{1}, \mathbf{u}_{1}\right)$ satisfies the equilibrium equations, in other words if the associated rest measure is zero (or, equivalently, if its norm is less than a fixed threshold), the next, and not so far from the previous, equilibrium point $\left(\lambda_{1}+\Delta \lambda, \mathbf{u}_{1}+\Delta \mathbf{u}\right)$ can be computed by means of the iterative scheme, based on the first-order approximation of the rest $\mathbf{s}\left(\mathbf{u}_{1},\left(\lambda_{1}+\Delta \lambda\right) \overline{\mathbf{u}}\right)(\Delta \lambda$ and $\Delta \mathbf{u}$ are the increments of $\lambda$ and $\mathbf{u}$, respectively), in formula

$$
\Delta \mathbf{u}=-\mathbf{K}^{-1} \mathbf{s}\left(\mathbf{u}_{1},\left(\lambda_{1}+\Delta \lambda\right) \overline{\mathbf{u}}\right)
$$

where we used the slim notation

$$
\mathbf{s}\left(\mathbf{u}_{1},\left(\lambda_{1}+\Delta \lambda\right) \overline{\mathbf{u}}\right)=\mathbf{s}\left(\left[\begin{array}{c}
\mathbf{u}_{1} \\
\left(\lambda_{1}+\Delta \lambda\right) \overline{\mathbf{u}}
\end{array}\right]\right)
$$

specifying that the reaction $\mathbf{s}$, even if we use the same symbol, collects only the terms correlated, in the sense of virtual work, with the unknown displacements collected in $\mathbf{u}$. The stiffness matrix $\mathbf{K}$, that is the Hessian of the total strain energy, is the part of the tangent stiffness matrix related to the unknown displacements, ie, that collected in $\mathbf{u}$, computed in $\left(\lambda_{1}, \mathbf{u}_{1}\right)$. As for the reaction $\mathbf{s}$, also the stiffness matrix can be assembled building the elementary contributions related to a pair of interacting grains.

It is well-known that the main drawback of Newton's scheme, see Equation 8, is related to the equilibrium points corresponding to quasi-singularity of the tangent stiffness matrix. Indeed, for these points, the convergence cannot be reached or becomes very slow. A brilliant idea to overcome this problem is due to Riks, which in 1979 introduced an arc-length strategy, see Riks. ${ }^{24}$ In other words, Riks suggested the idea that the equilibrium path can be described by using as parameter the arc-length of the curve instead of the nondimensional parameter $\lambda$ used to describe the external load.

A detailed description of arc-lenght method and its several variations can be found in Wriggers, ${ }^{25}$ while an efficient implementation of the arc-length iterative scheme is described in Turco and Caracciolo ${ }^{18}$ for plasticity problems and in Turco ${ }^{16}$ and Turco et $\mathrm{a}^{26}$ for elasticity problems considering large displacements. The interested readers can find a detailed description and some technicalities in the foregoing references. In the following, we only describe on the fly some computational details used in the in-house built code.

An elementary way for introducing the method is to impose that the linear extrapolation of the rest is zero:

$$
\mathbf{r}\left(\mathbf{u}_{1}+\Delta \mathbf{u}+\dot{\mathbf{u}},\left(\lambda_{1}+\Delta \lambda+\dot{\lambda}\right) \overline{\mathbf{u}}\right) \approx \mathbf{s}\left(\mathbf{u}_{1}+\Delta \mathbf{u},\left(\lambda_{1}+\Delta \lambda\right) \overline{\mathbf{u}}\right)+\mathbf{K}_{\mathbf{u u}} \dot{\mathbf{u}}+\mathbf{K}_{\mathbf{u} \overline{\mathbf{u}}} \dot{\lambda} \overline{\mathbf{u}}=\mathbf{0},
$$

where $\dot{\lambda}$ and $\dot{\mathbf{u}}$ define the correction corresponding to the extrapolation $\lambda_{1}+\Delta \lambda$ and $\mathbf{u}_{1}+\Delta \mathbf{u}$ and $\mathbf{K}_{\mathbf{u u}}$ and $\mathbf{K}_{\mathbf{u} \overline{\mathbf{u}}}$ are the blocks of the stiffness matrix $\mathbf{K}$ related to the free $\mathbf{u}$ and given $\overline{\mathbf{u}}$ displacements computed in $\left(\lambda_{1}+\Delta \lambda, \mathbf{u}_{1}+\Delta \mathbf{u}\right)$.

We underline that the nondimensional load parameter $\lambda$ has to be considered as an unknown, and therefore an additional equation has to be added. This additional equation is a constraint equation between the extrapolation $(\Delta \lambda, \Delta \mathbf{u})$ and correction $(\dot{\lambda}, \dot{\mathbf{u}})$. Many choices are possible, here, we consider a rather general constraint of the form

$$
\left[\begin{array}{c}
\Delta \mathbf{u} \\
\Delta \lambda \overline{\mathbf{u}}
\end{array}\right]^{T} \mathbf{C}\left[\begin{array}{c}
\dot{\mathbf{u}} \\
\dot{\lambda} \overline{\mathbf{u}}
\end{array}\right]=0,
$$

being $\mathbf{C}$ a matrix that can be chosen, eg, to improve the convergence ratio or to make simpler the necessary calculations. ${ }^{\text {I }}$ Starting from Equation 10, the correction $\dot{\mathbf{u}}$ can be obtained from

$$
\dot{\mathbf{u}}=-\mathbf{K}_{\mathbf{u u}}^{-1} \mathbf{s}\left(\mathbf{u}_{1}+\Delta \mathbf{u},\left(\lambda_{1}+\Delta \lambda+\dot{\lambda}\right) \overline{\mathbf{u}}\right)
$$

Successively, by using Equation 11 in the case $\mathbf{C}=\mathbf{K}$, the correction $\dot{\lambda}$ is worth

$$
\dot{\lambda}=-\frac{\Delta \mathbf{u}^{T} \mathbf{K}_{\mathbf{u u}} \dot{\mathbf{u}}+\Delta \lambda \overline{\mathbf{u}}^{T} \mathbf{K}_{\overline{\mathbf{u}} \mathbf{u}} \dot{\mathbf{u}}}{\Delta \mathbf{u}^{T} \mathbf{K}_{\mathbf{u} \overline{\mathbf{u}}} \overline{\mathbf{u}}+\Delta \lambda \overline{\mathbf{u}}^{T} \mathbf{K}_{\overline{\mathbf{u}} \overline{\mathbf{u}}} \overline{\mathbf{u}}} .
$$

\footnotetext{
${ }^{\pi}$ Equation 11 is a general form of the constraint equation, which is equivalent to imposing the arc-length (which is the parameter used in the Riks' description of the equilibrium path). In particular, it imposes a type of orthogonality between extrapolation $(\Delta \lambda, \Delta \mathbf{u})$ and correction $(\dot{\lambda}, \dot{\mathbf{u}})$, which can generate different algorithms on the basis of the particular choice of $\mathbf{C}$. The original version proposed by Riks, for example, considers the case $\mathbf{C}=\mathbf{I}$ where $\mathbf{I}$ is the identity matrix. Here, we used a slightly different and effective form which is, in our opinion, more convenient from the computational point of view.
} 
Equations 12 and 13 can be particularised choosing as $\mathbf{C}$ the matrix $\mathbf{K}$ in such a way that a very simple expression for the correction $\dot{\lambda}$ is:

$$
\dot{\lambda}=\frac{\Delta \mathbf{u}^{T} \mathbf{s}+\Delta \lambda \overline{\mathbf{u}}^{T} \mathbf{K}_{\overline{\mathbf{u}} \mathbf{u}} \mathbf{K}_{\mathbf{u} \mathbf{u}}^{-1} \mathbf{s}}{\Delta \lambda \overline{\mathbf{u}}^{T}\left(\mathbf{K}_{\overline{\mathbf{u}} \overline{\mathbf{u}}}-\mathbf{K}_{\overline{\mathbf{u}} \mathbf{u}} \mathbf{K}_{\mathbf{u u}}^{-1} \mathbf{K}_{\mathbf{u} \overline{\mathbf{u}}}\right) \overline{\mathbf{u}}} .
$$

The algorithm briefly outlined in the foregoing requires the first extrapolation in each step. The simpler one consists in its evaluation from the results of the previous steps. In formulae, using already defined quantities, we can use

$$
\begin{aligned}
\Delta \lambda & =m\left(\lambda_{1}-\lambda_{0}\right), \\
\Delta \mathbf{u} & =m\left(\mathbf{u}_{1}-\mathbf{u}_{0}\right),
\end{aligned}
$$

being the adaptive coefficient $m$ used to modify the arc-length during the stepwise procedure and $\left(\lambda_{1}, \mathbf{u}_{1}\right)$ and $\left(\lambda_{0}, \mathbf{u}_{0}\right)$ the last and the second last computed point of the equilibrium path, respectively. In practice, the adaptive coefficient $m$ increases the step-length in the parts of the equilibrium path that are quasi-linear and decreases the step-length in the parts of the equilibrium path that are highly nonlinear. A simple formula for computing the adaptive coefficient $m$, which increases the efficiency of the algorithm, see, Wriggers ${ }^{25}$ and Clarke and Hancock ${ }^{27 \#}$ is

$$
m=1-\frac{r_{l}-n_{l}}{r_{l}+n_{l}}
$$

which uses for modifying the arc-length the number of required loops $r_{l}$, computed by the code as the number of loops necessary to reach the convergence in the current step and the needed loops $n_{l}$ ( $n_{l}=5$ is the usual choice). For the first step, we can assume $m=1$, whereas the value of $\Delta \lambda$ is suitably estimated, fixing implicitly in this way the arc-length when the corresponding value of $\Delta \mathbf{u}$ is computed.

\section{4 | NUMERICAL SIMULATIONS AND THEIR INTERPRETATION}

The code developed following the guidelines presented in the foregoing section was applied to perform a series of numerical simulations. As a first step, we have chosen three different assemblies of grains, which can be regarded simultaneously as representative of two different modeling situations and options. Indeed, the number of involved grains is large enough that it is conceivable to introduce a continuum model for the whole assembly in which a material point models the behaviour of few grains, say a group of between 4 and 7 .

In a series of papers (see previous studies ${ }^{17,28-33}$ ), it has been proven that, rather unexpectedly, even in presence of relatively few degrees of freedom, discrete systems show a very similar behaviour to their continuous homogenised counterparts. However, we wanted to include a rather numerous set of grains (about 900) to make the continuous identification of the whole assembly more credible. On the other hand, we are aware of the fact that it is still very difficult (if not impossible) to consider large assemblies of grains including billions of grains. "Therefore, it is useful to consider the set of grains, which we have introduced as a representative elementary volume (REV), which, after homogenisation, may become a material point of a generalised continuum. It is clear that we do not know how to get a homogenised continuum model from our discrete granular system by using standard procedures. The results show that we will simply have a series of paradoxes if we impose ad hoc that such a homogenised model be of the kind given by Cauchy. For example, the results show the existence of boundary layers. We know by experience that the order of magnitude of the thickness of boundary layers is related to the internal length scales arising from stiffnesses. So, we expect to have at least two kinds of boundary layers eventually overlapping: those given by the length scales $L_{b a}$ and $L_{b c}$. However, a rigorous proof of this statement needs further investigations.

The criteria with which we have chosen the two loading tests and the three assemblies of grains is the following:

1. We want to show that one cannot introduce a standard Cauchy continuum to describe the behaviour of both the REV as a material particle and the considered assembly of grain as a continuous system.

2. We want to show that a small imperfection in the assembly may have remarkable effects on its global behaviour.

\footnotetext{
\# Although, formula (16) is not stated explicitly in ${ }^{25}$ and Clarke and Hancock ${ }^{27}$, in Clarke and Hancock ${ }^{27}$ (page 1374, equation (44) and following), there are suggestions, which used exactly the same quantities, that is the required $r_{l}$ and needed $n_{l}$ loops for the convergence; also in Wriggers ${ }^{25}$ (page 167), there is a suggestion without stating an explicit formula.

"However, we believe that up to millions of grains the principles of coding, which we have used may lead, with suitable modifications, to successful simulations. In any case, we are still very far from the number of grains imagined by Archimedes in his Sand Reckoner $\psi \alpha \mu \mu l \tau \eta \zeta$, Psammites).
} 
The preliminary results, which we present here, are suggestive. Indeed:

1. We prove that contact interactions to be introduced in the homogenised continuum model cannot be reduced to forces per unit area but must include contact couples.

2. We remark that to the microscopic kinematical descriptor of rotation of the single grain, a relevant deformation energy is associated and, as a consequence, to this descriptor, one cannot unequivocally associate the macro-descriptor of displacement; therefore, a corresponding extra-kinematical descriptor must be introduced, so excluding the possibility to use the Cauchy continuum model; instead at least a Cosserat continuum model has to be considered. Moreover, the following remarks are noteworthy: (i) it is clear from the proposed model that the rota-

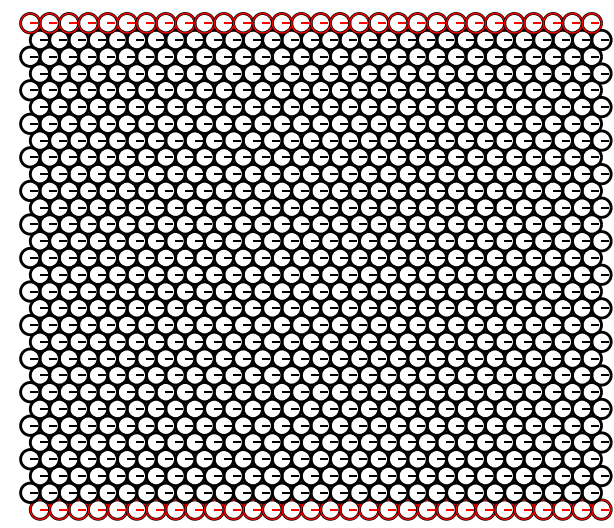

(A) without defects

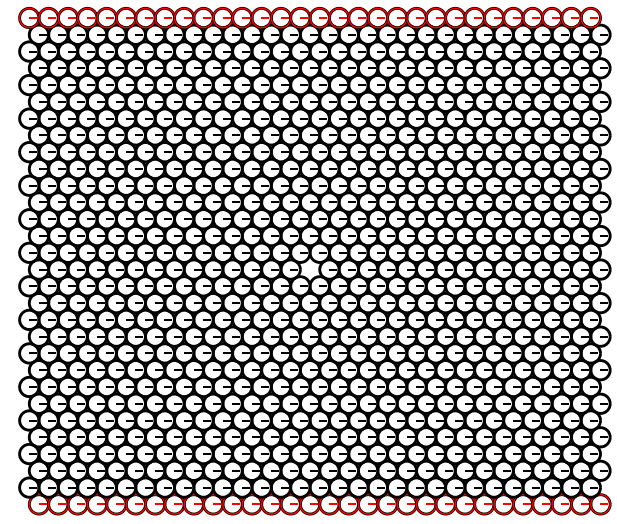

(B) one small defect

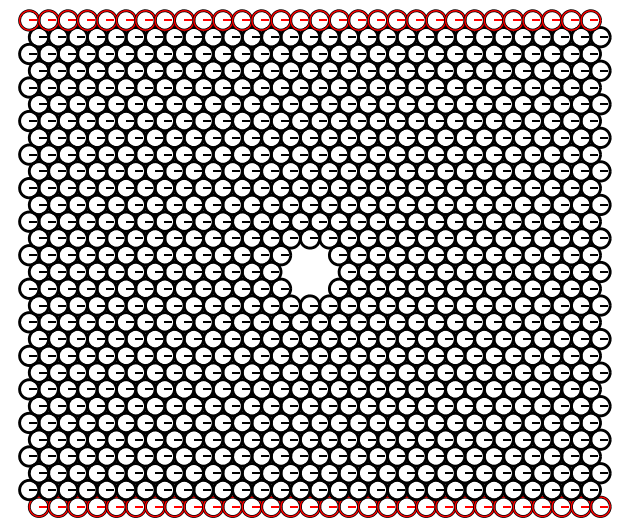

(C) one large defect

FIGURE 4 Regular packages of grains A, without defects; B, with one small defect; and C, with one large defect; on the red grains there are the assigned displacements [Colour figure can be viewed at wileyonlinelibrary.com] 
tions are independent variables with respect to placement in the discrete model for grains; (ii) in Cauchy's continua, the only possibility is to link rotations to the gradient of placement, and therefore these two kinematical quantities are not independent; (iii) there is no possibility to apply rotations of grains to the Cauchy's continuum model on the boundary, as in Cauchy's model, only placements (never gradients of placements) can be assigned on the boundary; (iv) there is no possibility in Cauchy's materials to have highly concentrated placement gradients close to the boundary in finite thickness layers.

3. We suggest that inside the assembly of grains, it is possible to distinguish the onset of boundary layers, when a suitable length scale, determined by the mechanical characteristic of the inter granular interactions, is sufficiently large compared with the grains dimensions; this length scale does not depend on the grain dimension and therefore will arise at macro-level after homogenisation.

Figure 4 shows the three regular assemblies, which we have considered. The first assembly is completely regular with the shift between one horizontal row and the closest one of half the diameter of a grain. The second assembly is like the first with a single grain vacancy in exactly the centre of the central row. The third assembly has a central hole constituted by removing three grains from the centre of the central row and two grains from each of the contiguous rows. The rows in red are those on which there is an imposed displacement. The row at the bottom has grains whose displacements and rotations are fixed. To the row at the top three, possible kinematical conditions are imposed:

1. Zero displacements and progressively increasing uniform rotations, until 0.4 rad (that is approximatively $23^{\circ}$ ) are assigned;

2. Imposed horizontal uniform displacement, until $2.5 D_{g}$ being $D_{g}$ the diameter of each one grain, is assigned with free rotations;

3. Imposed linear vertical displacement, from $1.5 D_{g}$ (on the top-left) to $3 D_{g}$ (on the top-right), is assigned with free rotations.

We combine the triplets of stiffness $a, b$, and $c$, see (1), into two length scales

$$
\begin{aligned}
L_{b a} & =\sqrt{\frac{b}{a}}, \\
L_{b c} & =\sqrt{\frac{b}{c}} .
\end{aligned}
$$

These length scales are useful for comparing the values of the resultant $R_{1}$ of the forces $f_{1}$, see Figure 3 , and the resultant $R_{2}$ of the forces $f_{2}$ applied to all grains at the bottom. For the total couple of these grains, we have used the auxiliary resultant force $R_{3}$. We calculate for each grain the intensity $f_{3}$ and then obtain $R_{3}$ as a sum of these intensities over all the bottom grains.

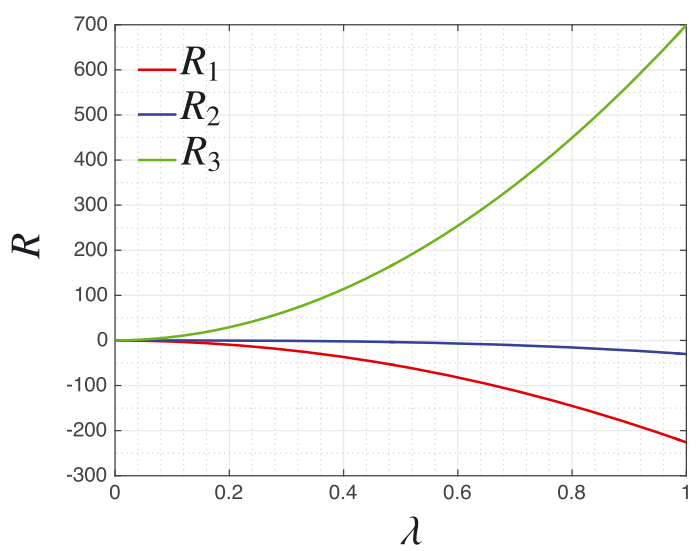

(A) $L=10$

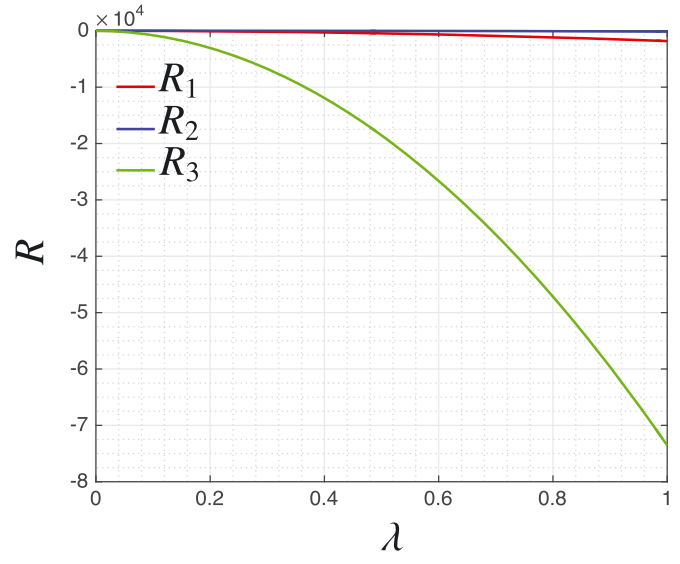

(B) $L=100$

FIGURE 5 Regular packages of grains without defects under given rotations: reactions on the bottom side varying $\lambda$ when A, $L=10$ and $\mathrm{B}, L=100$ [Colour figure can be viewed at wileyonlinelibrary.com] 


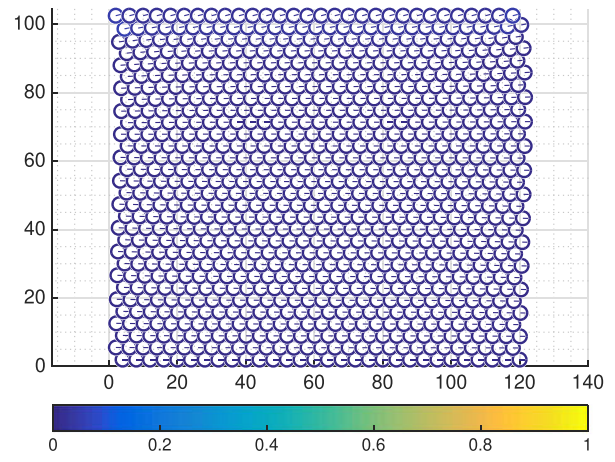

(A) $\lambda=0.5$, stretching energy

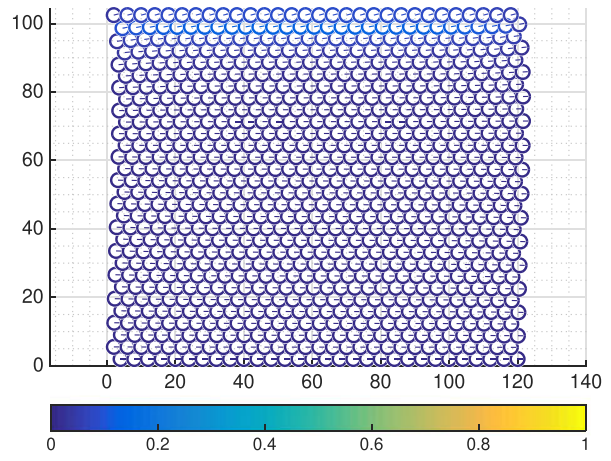

(C) $\lambda=0.5$, bending energy

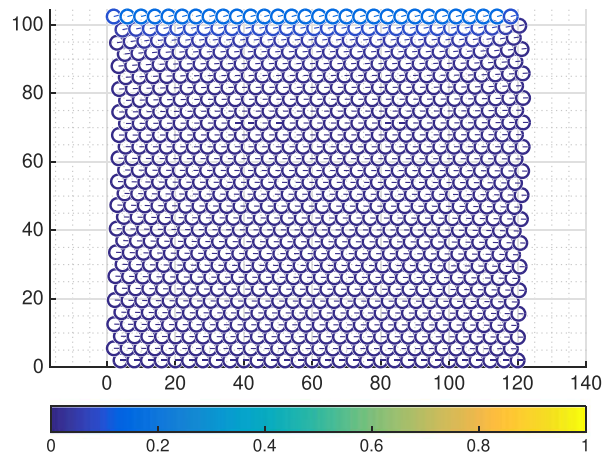

(E) $\lambda=0.5$, shearing energy

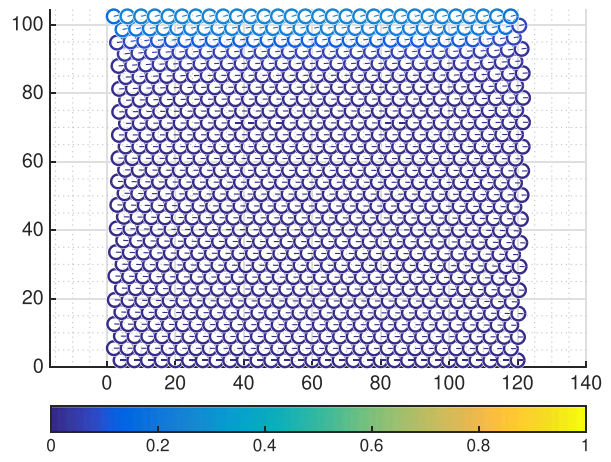

(G) $\lambda=0.5$, strain energy

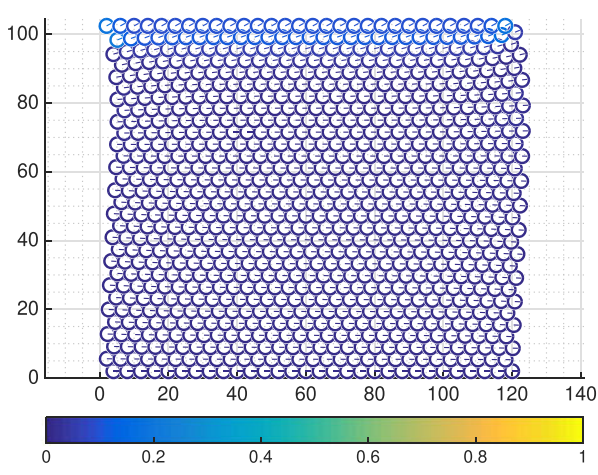

(B) $\lambda=1$, stretching energy

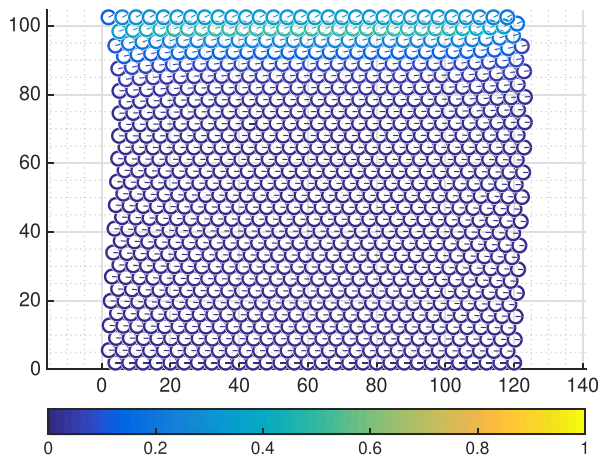

(D) $\lambda=1$, bending energy

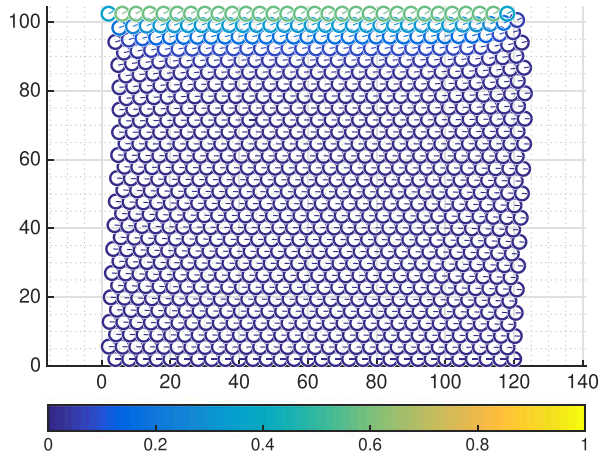

(F) $\lambda=1$, shearing energy

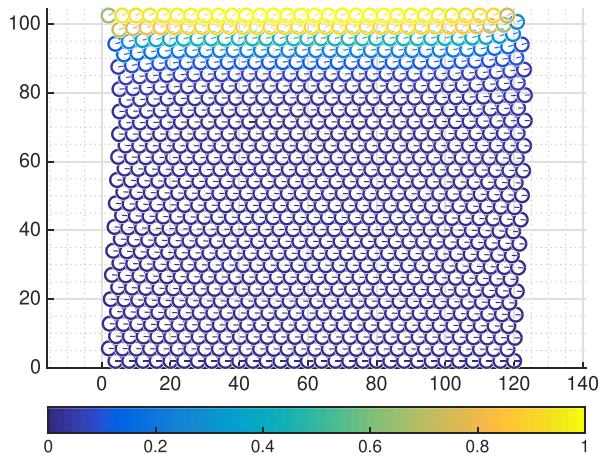

(H) $\lambda=1$, strain energy

FIGURE 7 Regular packages of grains without defects under rotations on the upside: deformations for $\lambda=0.5$ (on the left) and $\lambda=1$ (on the right) for $L=10$; colours show the density of the strain energy (stretching on the first row, bending on the second row, shearing on the third row, and their sum on the fourth row) [Colour figure can be viewed at wileyonlinelibrary.com] 


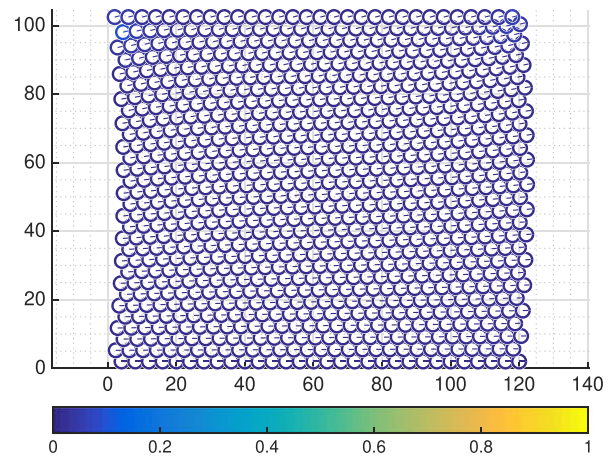

(A) $\lambda=0.5$, stretching energy

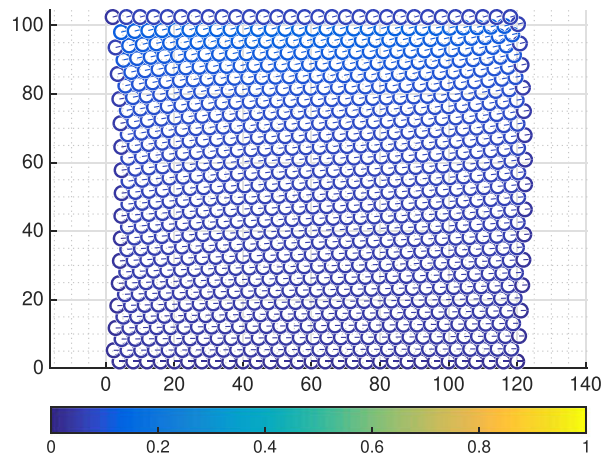

(C) $\lambda=0.5$, bending energy

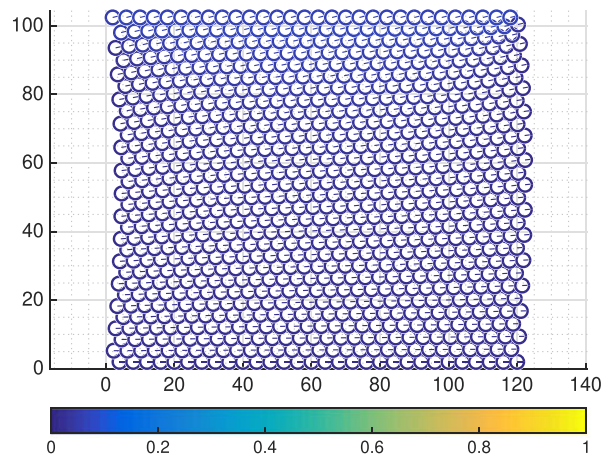

(E) $\lambda=0.5$, shearing energy

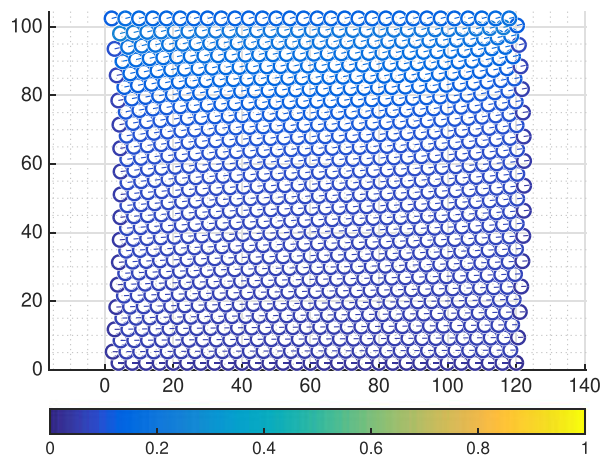

(G) $\lambda=0.5$, strain energy

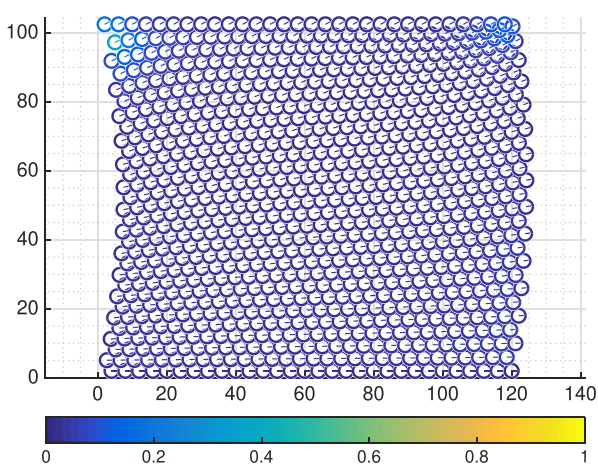

(B) $\lambda=1$, stretching energy

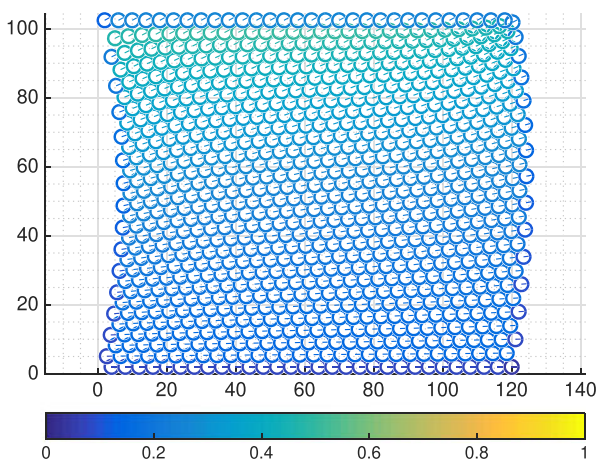

(D) $\lambda=1$, bending energy

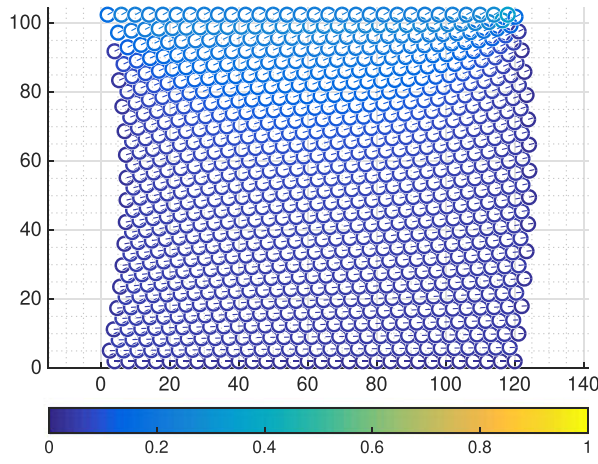

(F) $\lambda=1$, shearing energy

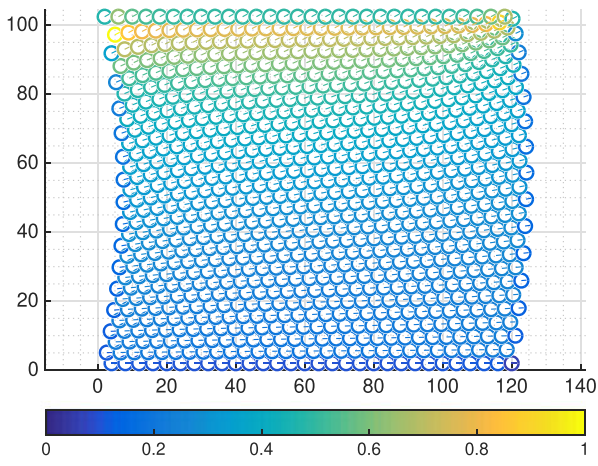

(H) $\lambda=1$, strain energy

FIGURE 8 Regular packages of grains without defects under rotations on the upside: deformations for $\lambda=0.5$ (on the left) and $\lambda=1$ (on the right) for $L=100$; colours show the density of the strain energy (stretching on the first row, bending on the second row, shearing on the third row, and their sum on the fourth row) [Colour figure can be viewed at wileyonlinelibrary.com] 
$L=10$ seems concentrated in the first few rows of grains at the top of the assembly. This boundary layer induces, however, a deformation pattern outside the layer itself. This pattern is better understood when zooming on the bottom last 10 row of grains. In Figure 8A, a clockwise pattern of rotation is shown to be typical of the deformation outside the boundary layer. Instead, when the boundary layer occupies the whole specimen, then the counterclockwise pattern is present in all the specimen.

\section{2 | Package with a small defect under given rotations and shear on the top row}

When we consider the package of grains with the small defect described in Figure 4B in both cases of imposed rotation and imposed shear displacement on the top row, we have recognised only a very localised and very small differences with the case of perfect assembly shown in Figure 4A. This circumstance indicates that, at least in the set of deformations states, which we have considered, the homogenised model to be introduced is a Cosserat continuum.

In these continua, applicable external actions include, together with surface forces, also contact couples. However, contact and volume forces concentrated on points are not possible. The existence of the imperfection has to be regarded as an internal boundary of the body (a kind of internal cut). In considered instance, this cut cannot be regarded as an internal surface of line cut but has to be modeled as a point. The effect of disconnected internal surface or line can be detected in Cosserat continua, while, on the contrary, the existence of an internal disconnection concentrated on a point does not affect the global deformation state.

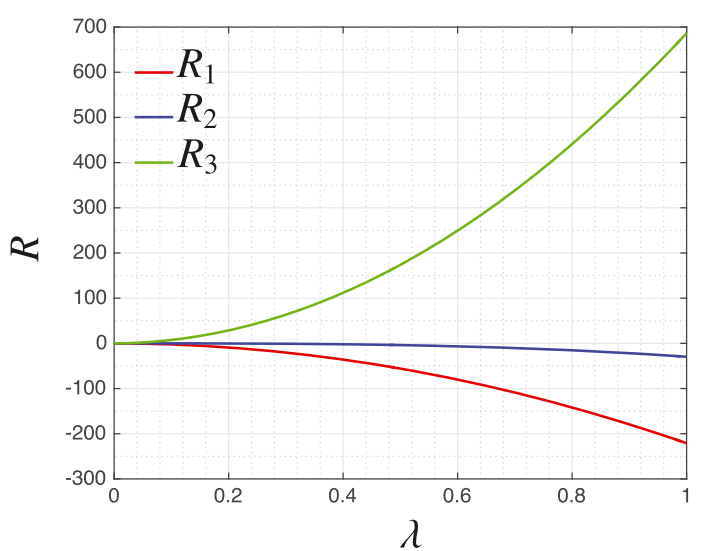

(A) $L=10$

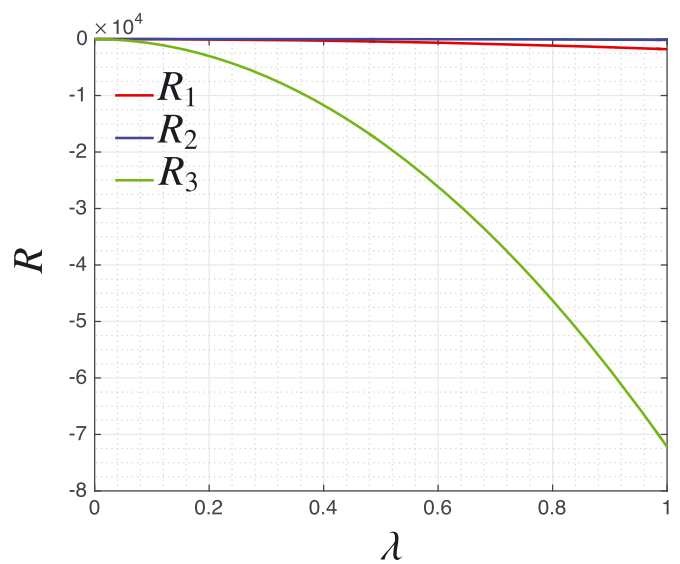

(B) $L=100$

FIGURE 11 Regular packages of grains with one large defect under given rotations on the upside: reaction on the bottom side varying $\lambda$ when A, $L=10$ and B, $L=100$ [Colour figure can be viewed at wileyonlinelibrary.com]

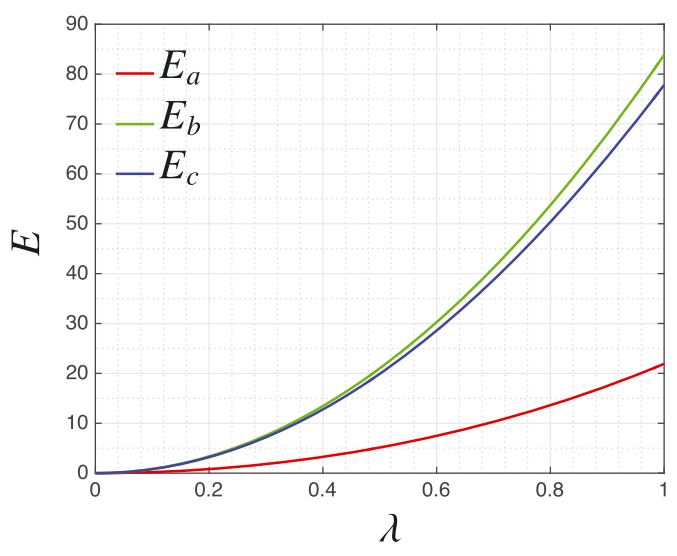

(A) $L=10$

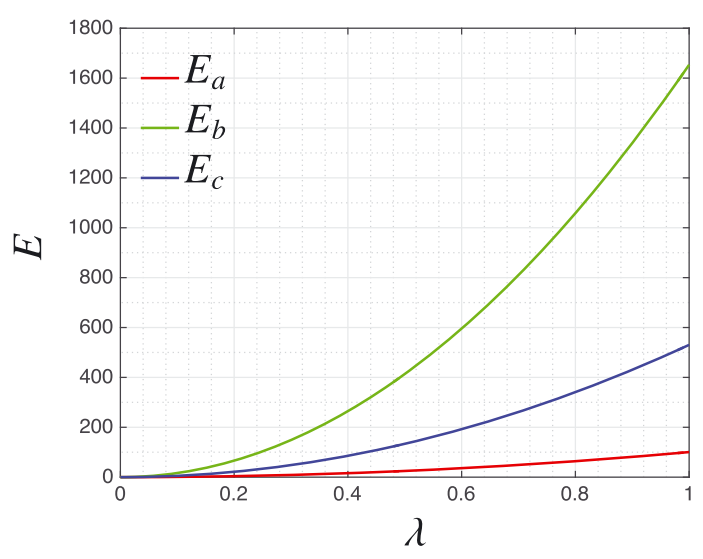

(B) $L=100$

FIGURE 12 Regular packages of grains with one large defect under given rotations: strain energies (stretching $E_{a}$, bending $E_{b}$, and shearing $E_{c}$ ) varying $\lambda$ when $\mathrm{A}, L=10$ and $\mathrm{B}, L=100$ [Colour figure can be viewed at wileyonlinelibrary.com] 


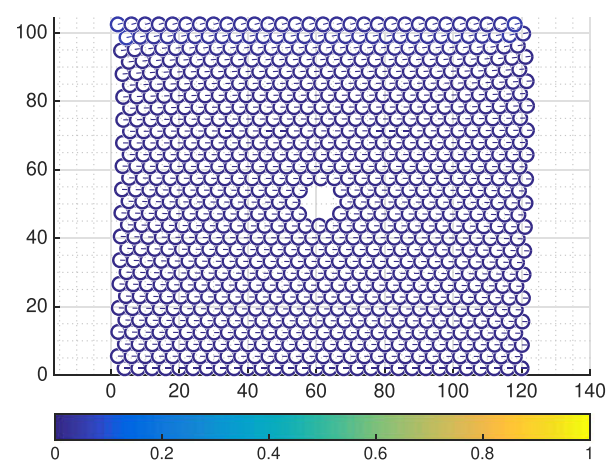

(A) $\lambda=0.5$, stretching energy

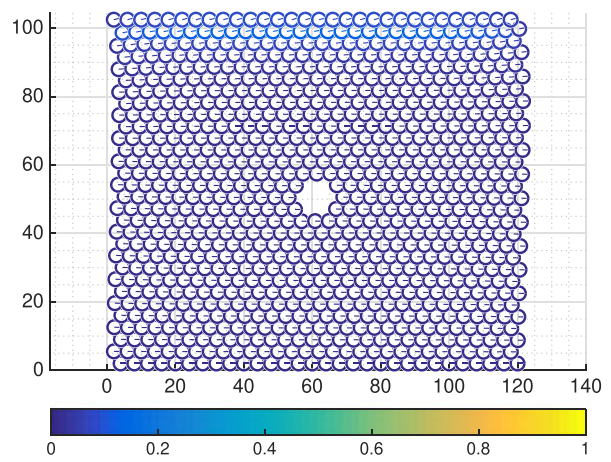

(C) $\lambda=0.5$, bending energy

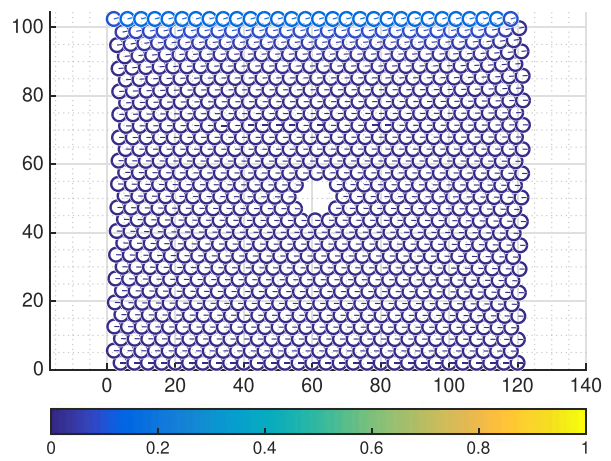

(E) $\lambda=0.5$, shearing energy

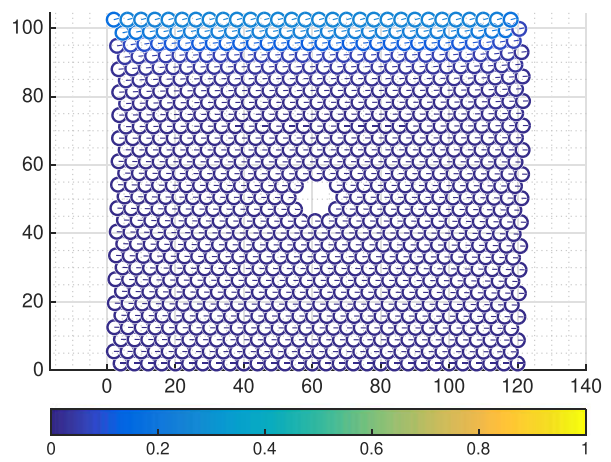

(G) $\lambda=0.5$, strain energy

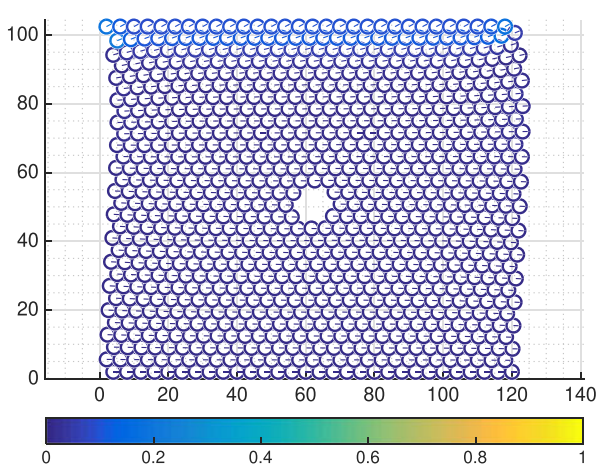

(B) $\lambda=1$, stretching energy

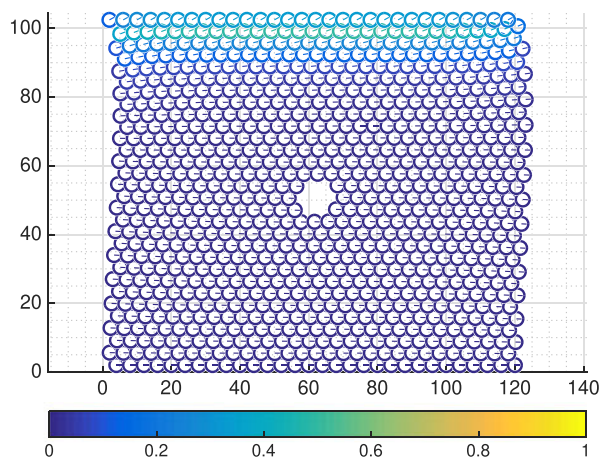

(D) $\lambda=1$, bending energy

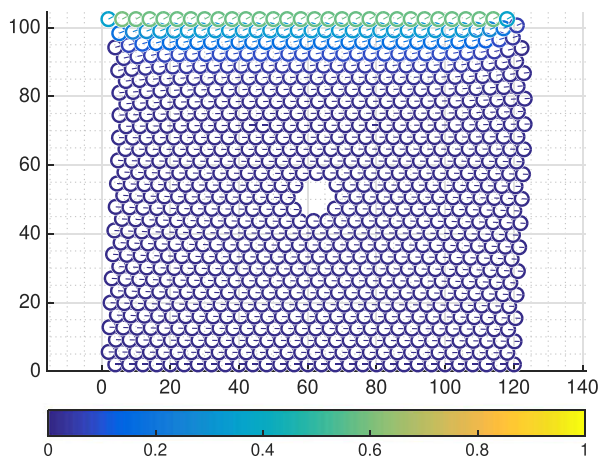

(F) $\lambda=1$, shearing energy

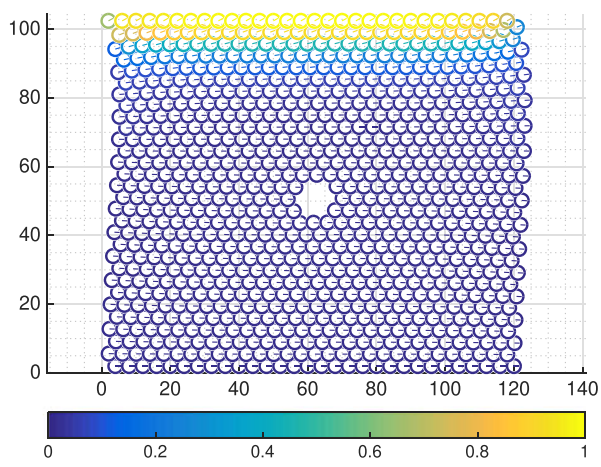

(H) $\lambda=1$, strain energy

FIGURE 13 Regular packages of grains with a large defect under rotations on the upside: deformations for $\lambda=0.5$ (on the left) and $\lambda=1$ (on the right) for $L=10$; colours show the density of the strain energy (stretching on the first row, bending on the second row, shearing on the third row, and their sum on the fourth row) [Colour figure can be viewed at wileyonlinelibrary.com] 


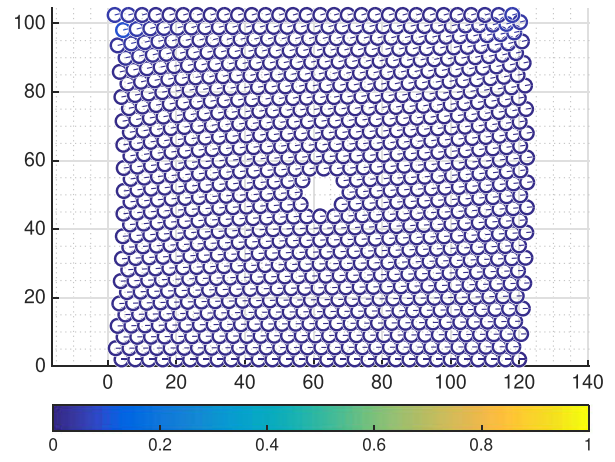

(A) $\lambda=0.5$, stretching energy

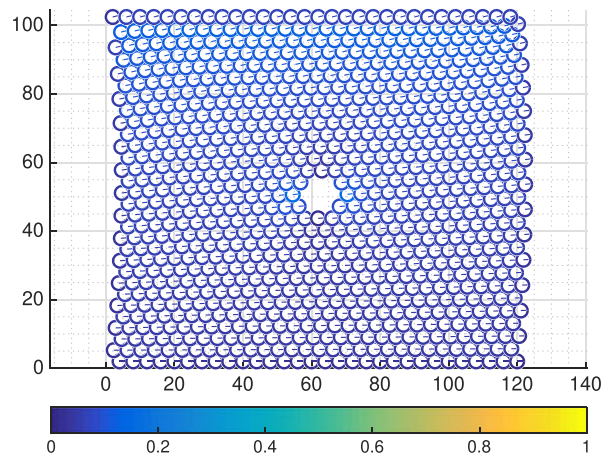

(C) $\lambda=0.5$, bending energy

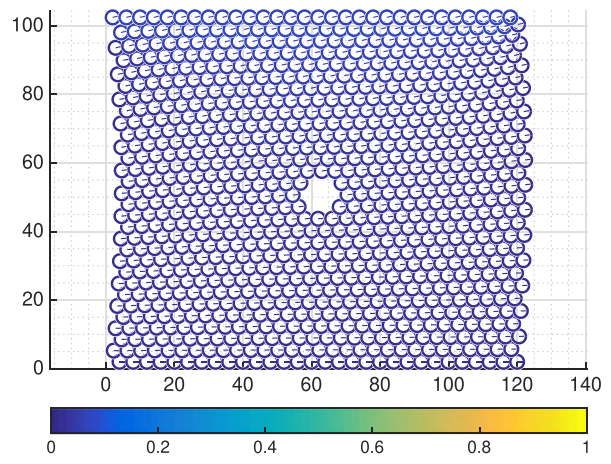

(E) $\lambda=0.5$, shearing energy

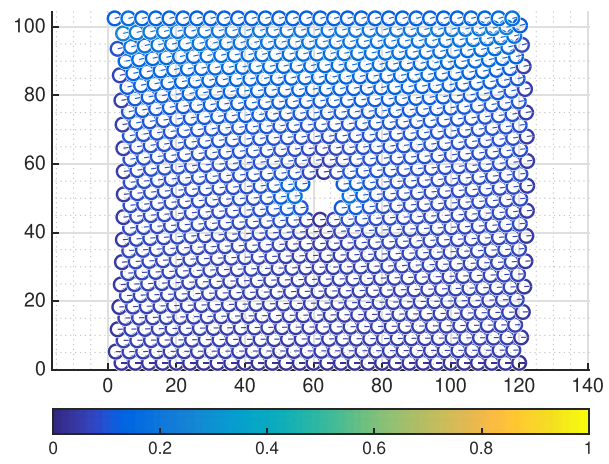

(G) $\lambda=0.5$, strain energy

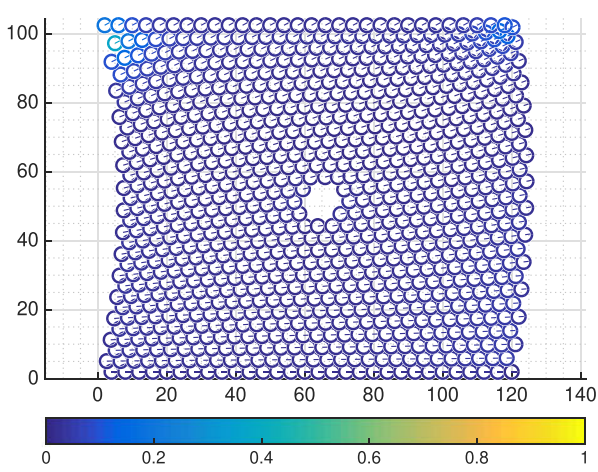

(B) $\lambda=1$, stretching energy

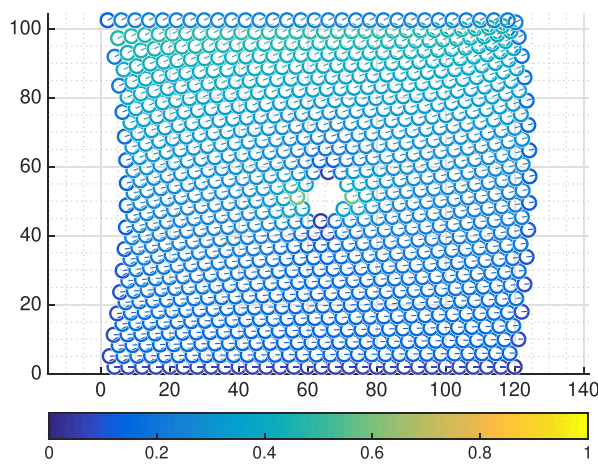

(D) $\lambda=1$, bending energy

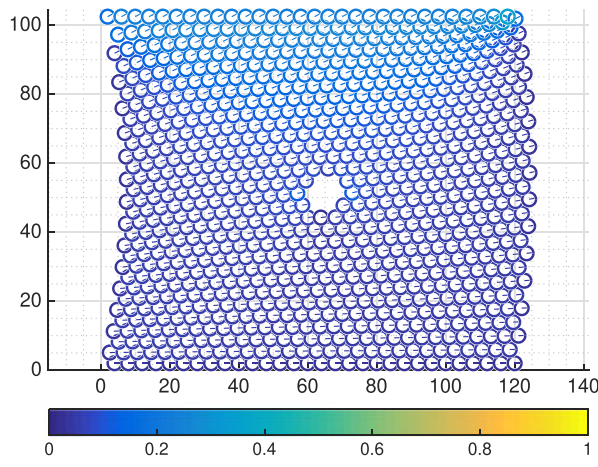

(F) $\lambda=1$, shearing energy

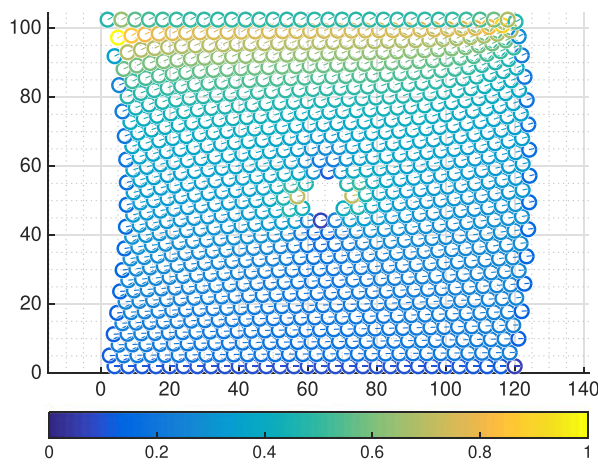

(H) $\lambda=1$, strain energy

FIGURE 14 Regular packages of grains with a large defect under rotations on the upside: deformations for $\lambda=0.5$ (on the left) and $\lambda=1$ (on the right) for $L=100$; colours show the density of the strain energy (stretching on the first row, bending on the second row, shearing on the third row, and their sum on the fourth row) [Colour figure can be viewed at wileyonlinelibrary.com] 
For saving space in the present paper, we do not reproduce the careful numerical simulation, which we have performed to substantiate the aforementioned statement. Actually, the reader can precisely refer to all the figures presented for the perfect assembly of grains. For more details about the performances, which one should expect from higher gradient and microstructures continua the reader is referred, eg, to previous studies. ${ }^{34-37}$

\subsection{Package with a large defect under given rotations on the top}

In the considered case, the internal hole, which introduces the assembly defect described by Figure 4C, is unloaded. This means that there are neither imposed rotations nor imposed displacements. We remark at first that the overall behaviour of the assembly, as a Cosserat continuum, does not change because of the introduction of the defect. Indeed (compare Figures 5 and 6 with Figures 11 and 12), the global response of the grains assembly seems not to be affected by the presence of the hole: All reactions $R_{1}, R_{2}$, and $R_{3}$, for both $L=10$ and $L=100$, and all values of deformation energies are qualitatively and quantitatively identical in presence and absence of holes. However, the internal cut is large enough to have an effect on the overall deformation patterns when $L=100$.

We start referring to Figure 13, which describes the case $L=10$. The boundary layer induced by the imposed rotations is limited to the first five rows and does not include the added hole. In fact, this boundary layer is very similar to the one presented in Figure 7. Therefore, as expected by heuristic considerations based on the study of boundary layers in phase transition problems (see, eg, dell'Isola et $\mathrm{al}^{38}$ ), in the case $L=10$, considering that the grains have length equal to four units, Cosserat type deformation cannot reach the depth of the hole, which is, in the considered units, appear in row 14,15,

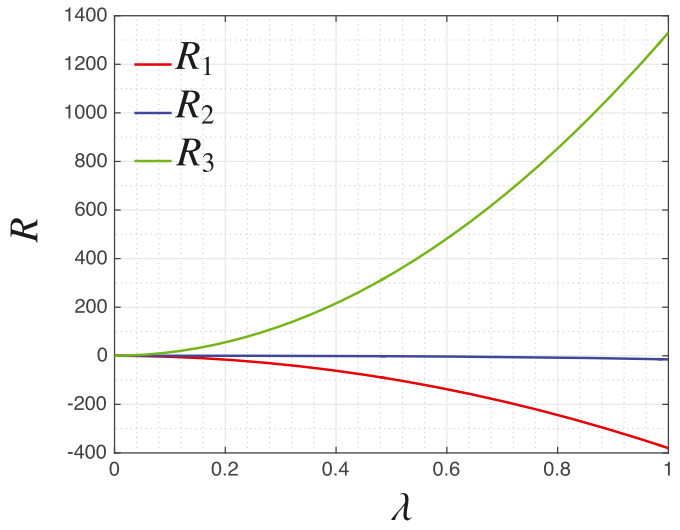

(A) $L=10$

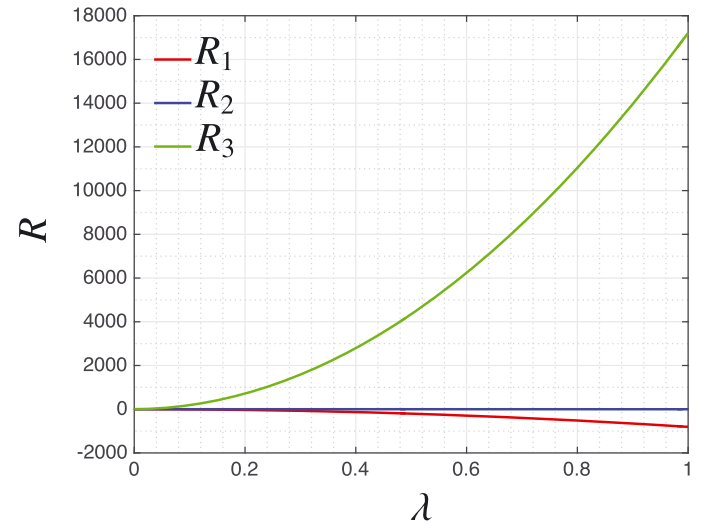

(B) $L=100$

FIGURE 15 Regular packages of grains without defects under shear on the upside: reaction on the bottom side varying $\lambda$ when $\mathrm{A}, L=10$ and $\mathrm{B}, L=100$ [Colour figure can be viewed at wileyonlinelibrary.com]

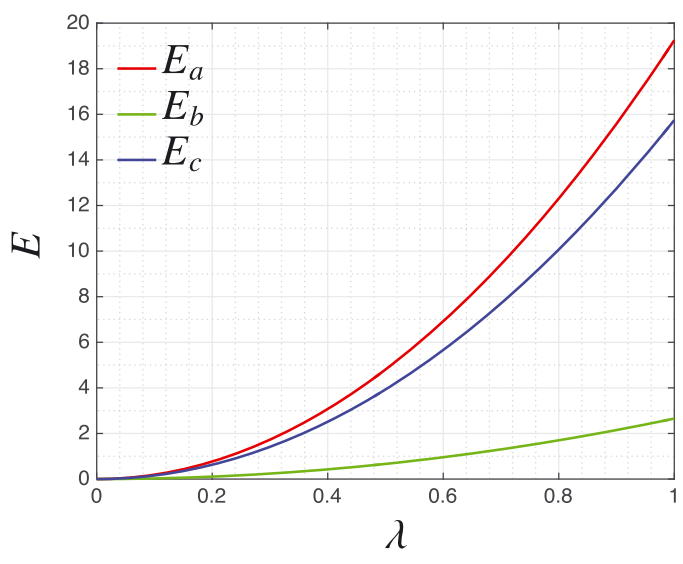

(A) $L=10$

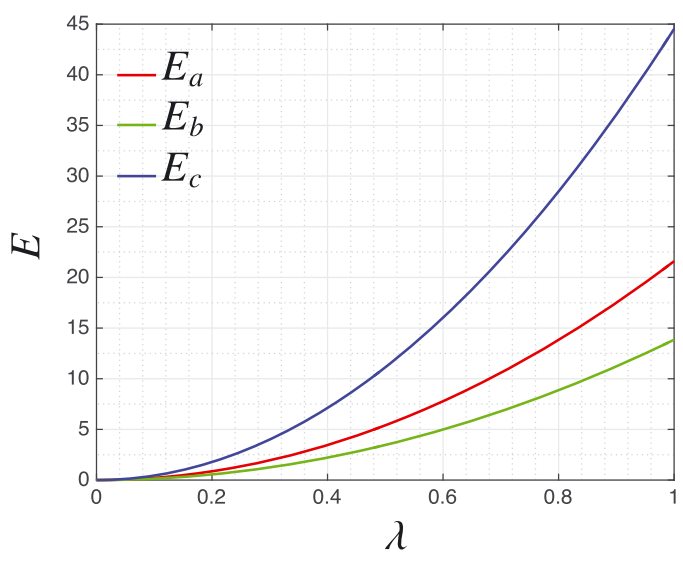

(B) $L=100$

FIGURE 16 Regular packages of grains without defects under shear: strain energies (stretching, bending, and shearing) varying $\lambda$ when A, $L=10$ and $\mathrm{B}, L=100$ [Colour figure can be viewed at wileyonlinelibrary.com] 
and 16 from the bottom. We now compare Figure 8 and Figure 14. It has to be remarked that the presence of the hole is producing, when $\lambda=1$, a kind of reflection and transmission pattern, which is rather suggestive. Instead, when $\lambda=0.5$ only barely perceptible (at the considered energy scale) differences between the case with and the case without hole can be observed. We remark the phenomenon of inversion of reaction couples, that is, the change of the sign of $R_{3}$, which was observe in the absence of holes is preserved (see Figure 11). Also, the bulge observed on the right of the deformation pattern, which is one of its peculiar features imposing a Cosserat description, is not influenced by the presence of the hole, see previous studies. ${ }^{39-41}$

\section{4 | Packages under a shear displacement on the top}

As clearly shown by Figure 15, in the case of imposed shear displacement at the top, the rotation inversion phenomenon observed in the case of imposed rotations at the top is not occurring. Also, in the case of imposed shear displacement, as in the case of imposed rotations, the reactions at the bottom, and the deformation energies (see Figure 16) as functions of $\lambda$, are not affected by the presence of the hole (compare Figures 15 and 16 with Figures 17 and 18). Concerning Figure 19, which considers the case $L=10$, we remark that the typical deformation pattern of shear deformed rectangular specimens is reproduced for the extensional energy in Figure 19B and for the shear energy in Figure 19F. On the contrary, bending energy has a space pattern with localised deformation energy at the bottom with the formation of a boundary layer, see Figure 19D.

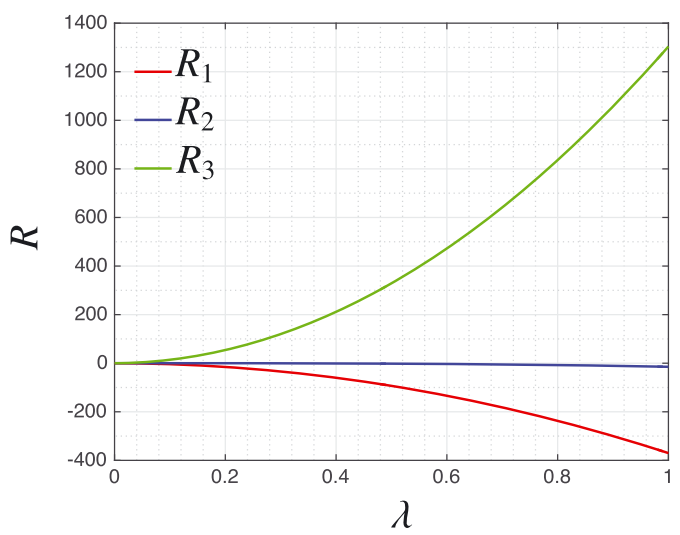

(A) $L=10$

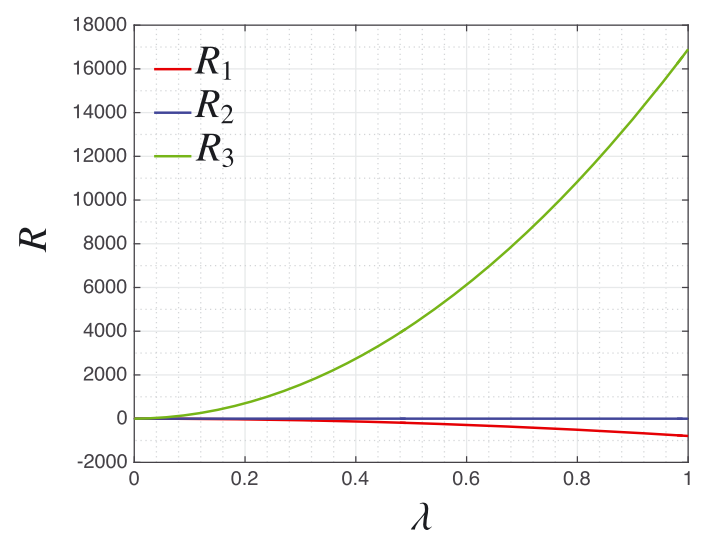

(B) $L=100$

FIGURE 17 Regular packages of grains with large defect under shear on the upside: reaction on the bottom side varying $\lambda$ when A, $L=10$ and $\mathrm{B}, L=100$ [Colour figure can be viewed at wileyonlinelibrary.com]

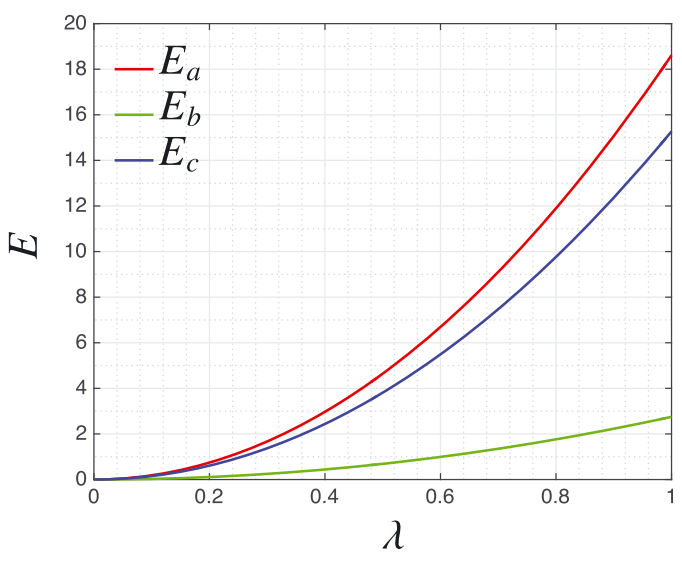

(A) $L=10$

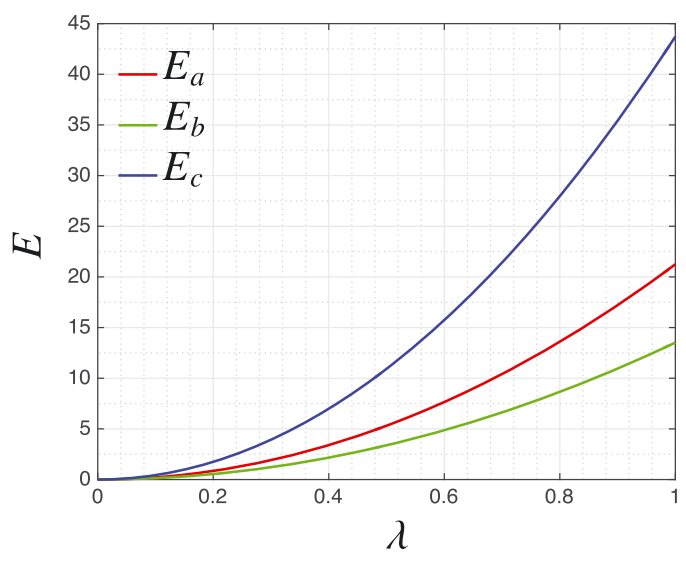

(B) $L=100$

FIGURE 18 Regular packages of grains with large defect under shear on the upside: strain energies (stretching, bending, and shearing) varying $\lambda$ when $\mathrm{A}, L=10$ and $\mathrm{B}, L=100$ [Colour figure can be viewed at wileyonlinelibrary.com] 


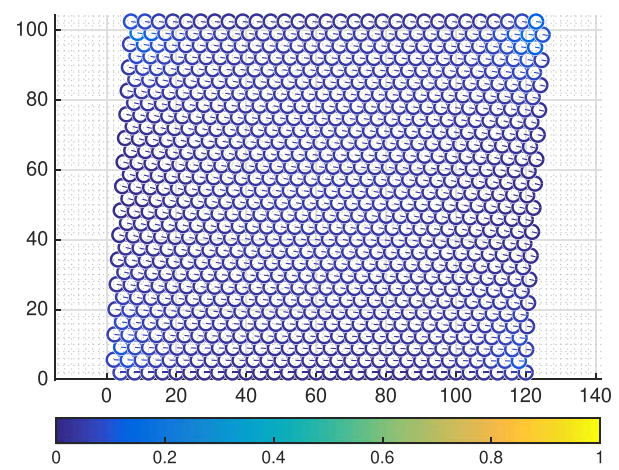

(A) $\lambda=0.5$, stretching energy

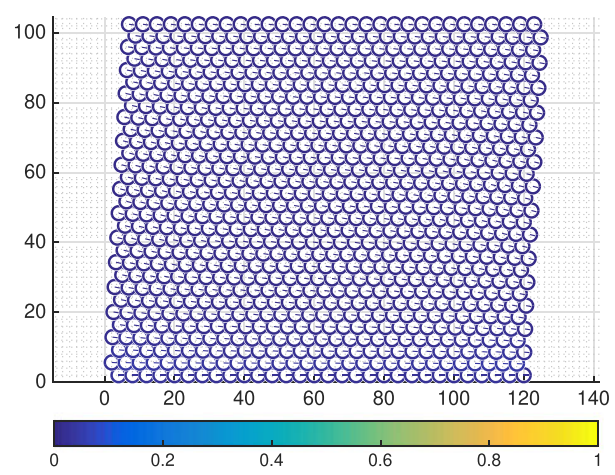

(C) $\lambda=0.5$, bending energy

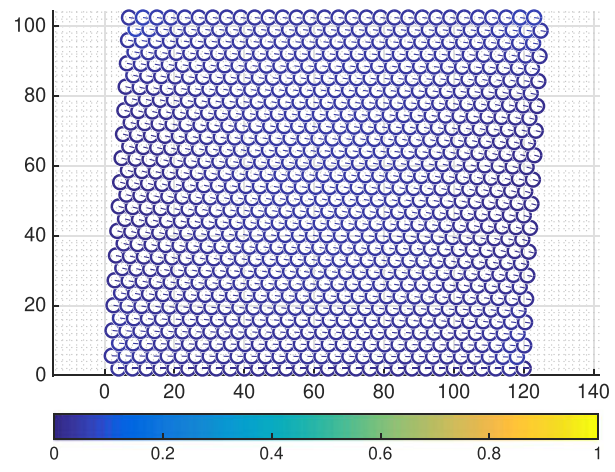

(E) $\lambda=0.5$, shearing energy

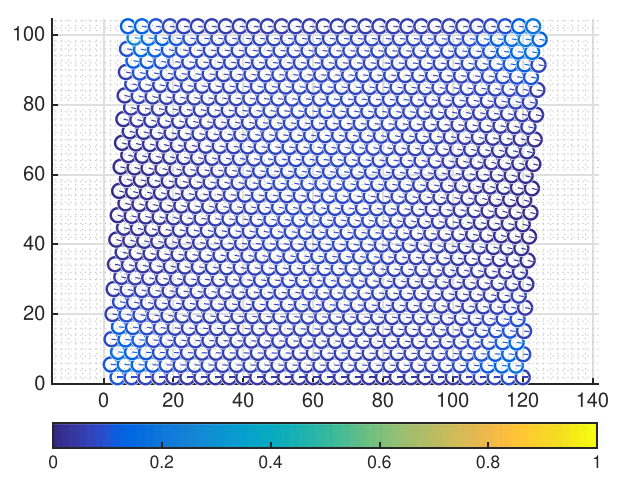

(G) $\lambda=0.5$, strain energy

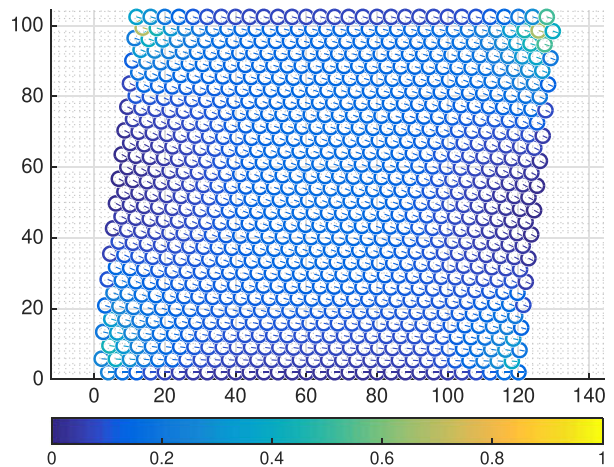

(B) $\lambda=1$, stretching energy

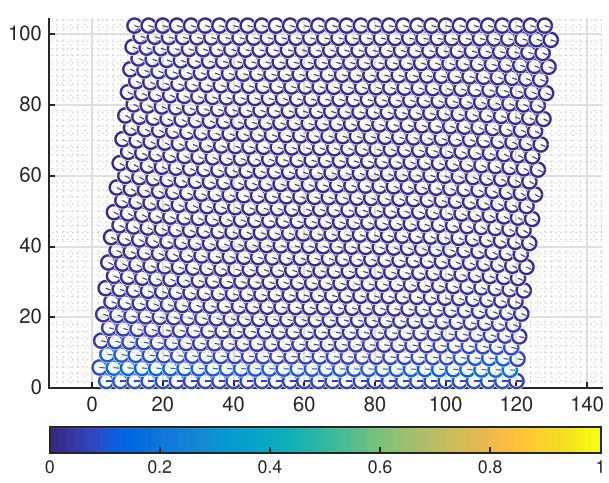

(D) $\lambda=1$, bending energy

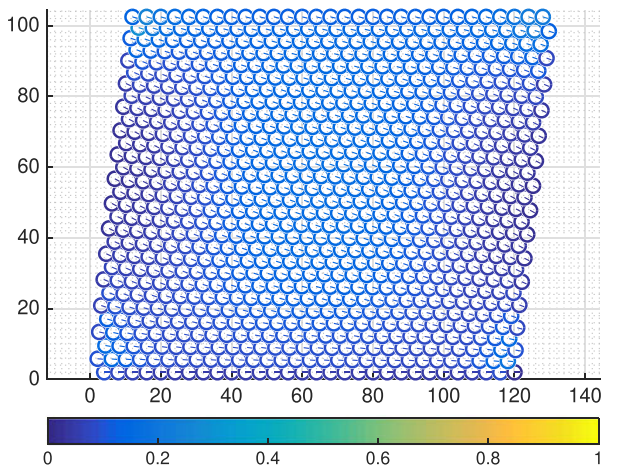

(F) $\lambda=1$, shearing energy

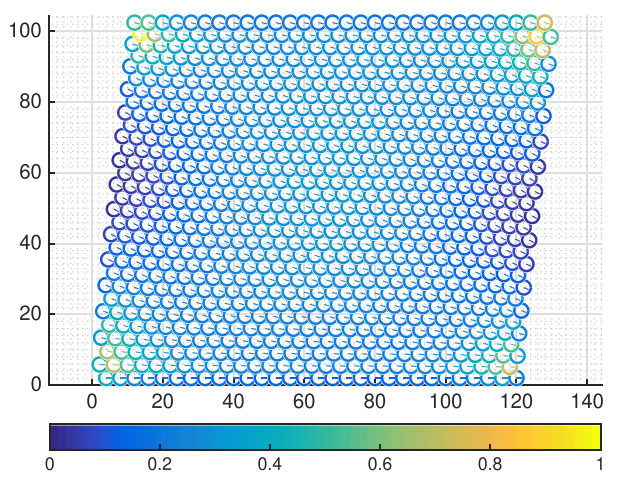

(H) $\lambda=1$, strain energy

FIGURE 19 Regular packages of grains without defects under shear on the upside: deformations for $\lambda=0.5$ (on the left) and $\lambda=1$ (on the right) for $L=10$; colours show the density of the strain energy (stretching on the first row, bending on the second row, shearing on the third row, and their sum on the fourth row) [Colour figure can be viewed at wileyonlinelibrary.com] 


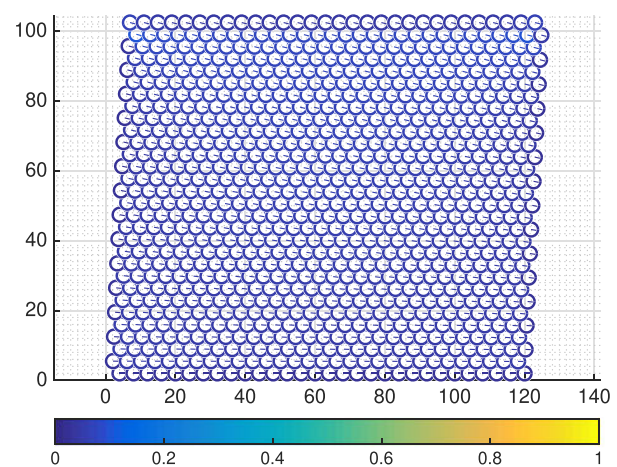

(A) $\lambda=0.5$, stretching energy

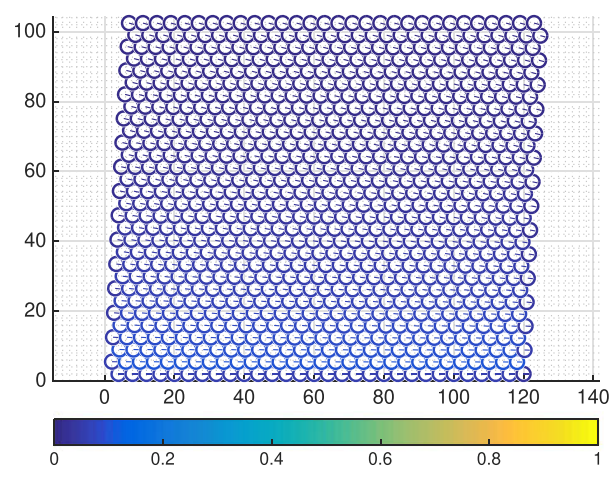

(C) $\lambda=0.5$, bending energy

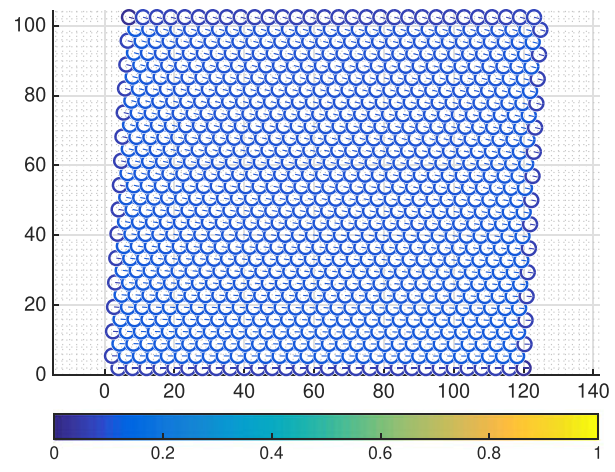

(E) $\lambda=0.5$, shearing energy

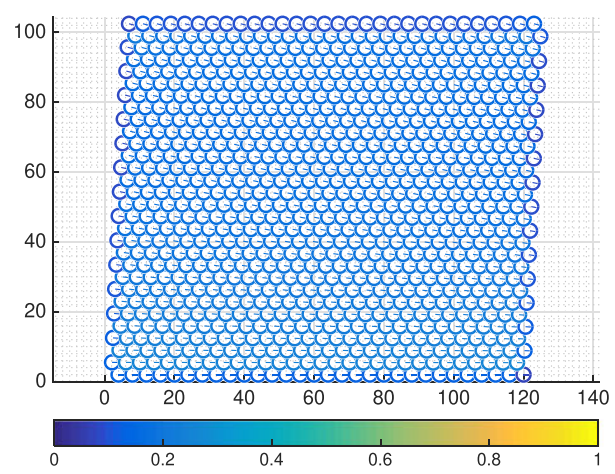

(G) $\lambda=0.5$, strain energy

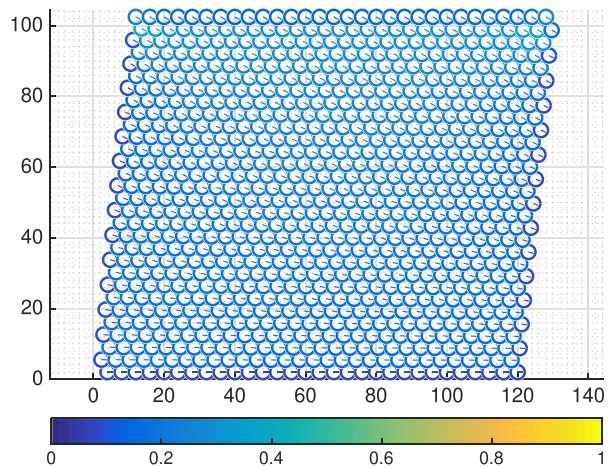

(B) $\lambda=1$, stretching energy

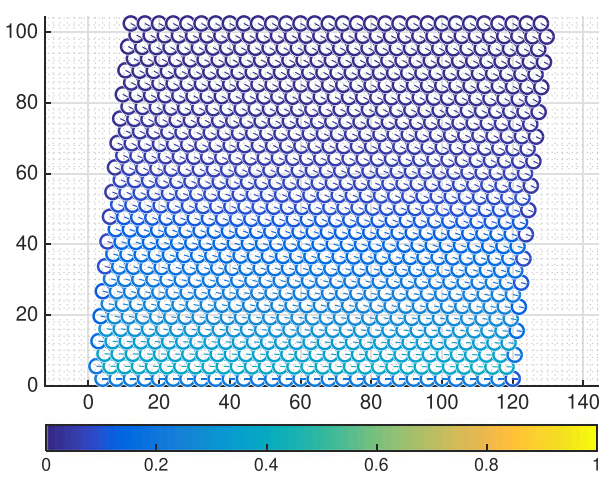

(D) $\lambda=1$, bending energy

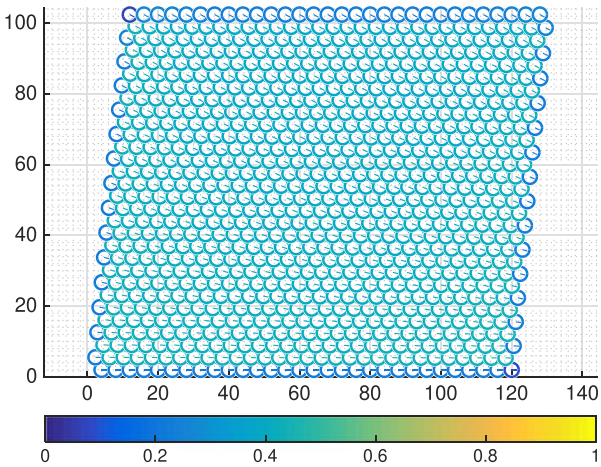

(F) $\lambda=1$, shearing energy

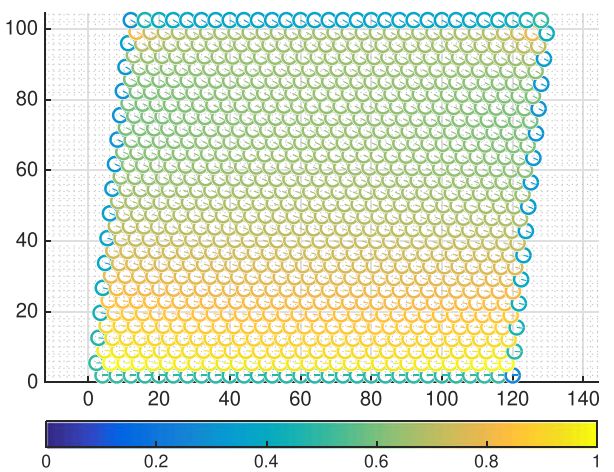

(H) $\lambda=1$, strain energy

FIGURE 20 Regular packages of grains without defects under shear on the upside: deformations for $\lambda=0.5$ (on the left) and $\lambda=1$ (on the right) for $L=100$; colours show the density of the strain energy (stretching on the first row, bending on the second row, shearing on the third row, and their sum on the fourth row) [Colour figure can be viewed at wileyonlinelibrary.com] 


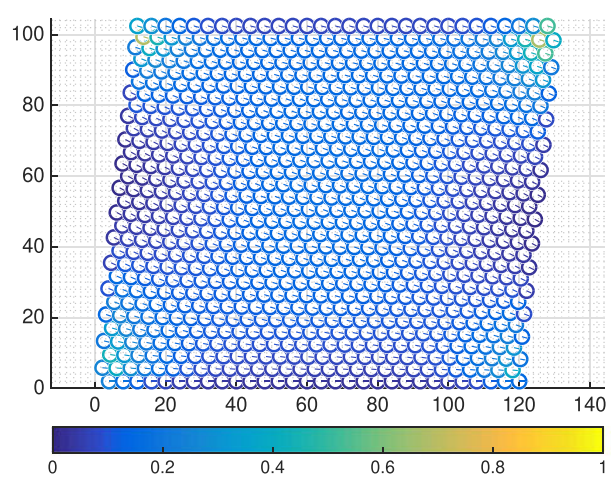

(A) streching energy

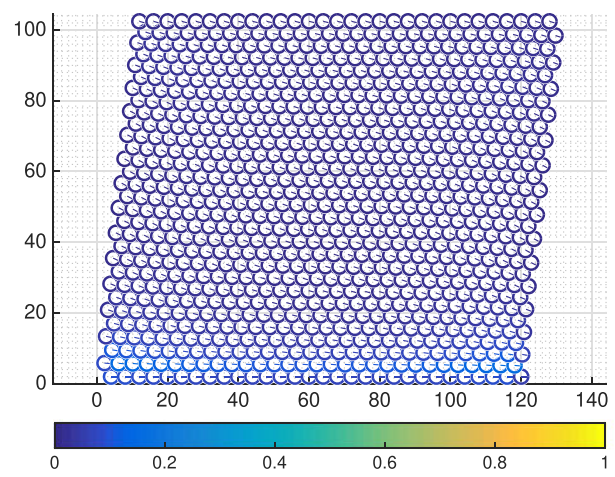

(C) bending energy

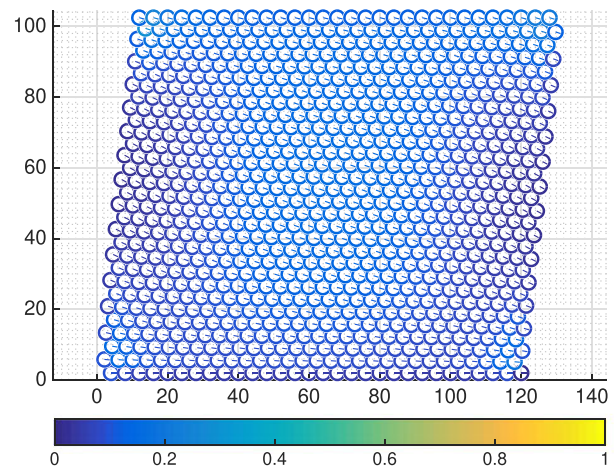

(E) shearing energy

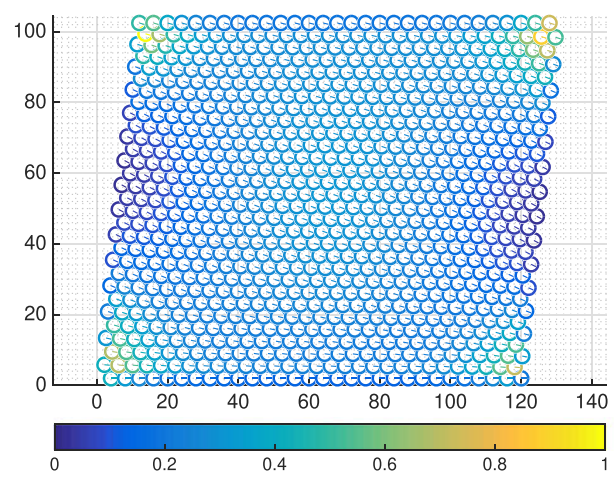

(G) strain energy

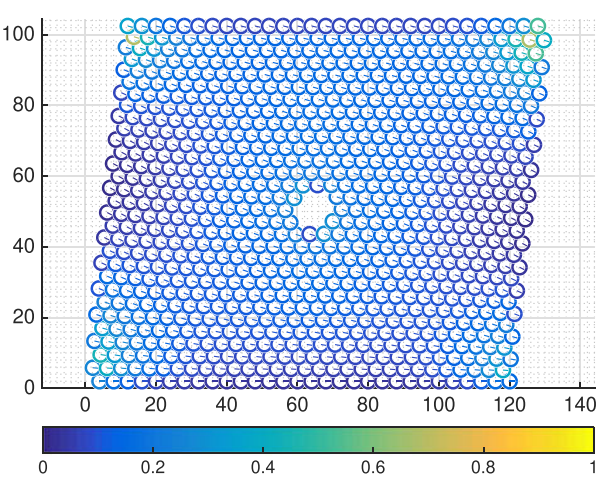

(B) streching energy

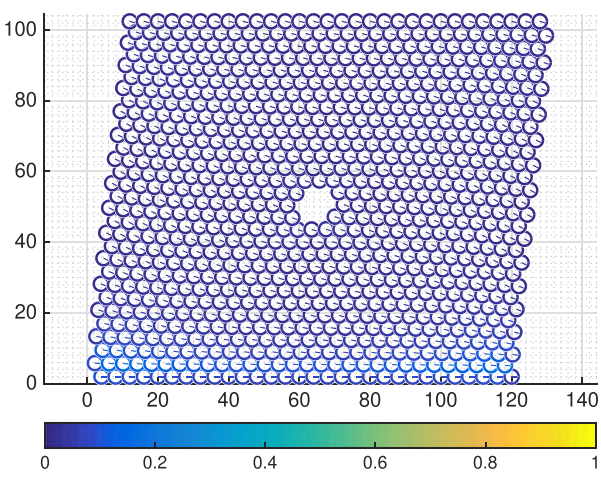

(D) bending energy

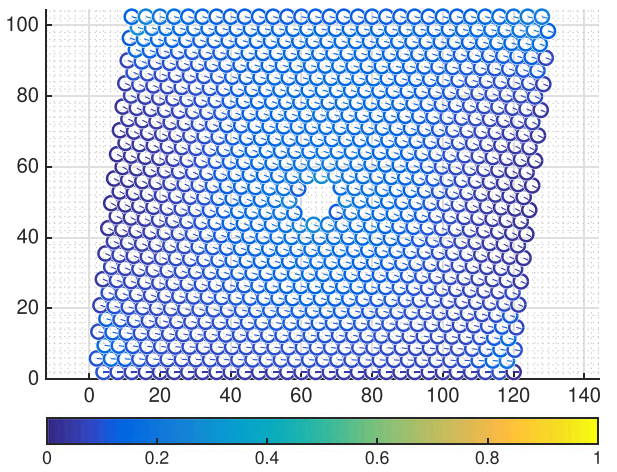

(F) shearing energy

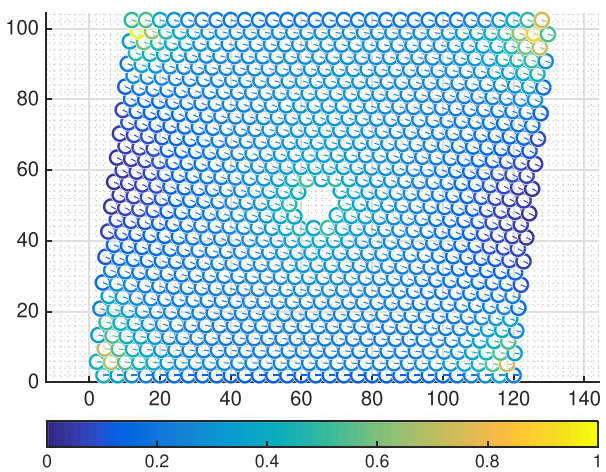

(H) strain energy

FIGURE 21 Regular packages of grains without (on the left) and with one large defect (on the right) under shear on the upside: deformations for $\lambda=1$ for $L=10$; colours show the density of the strain energy (stretching on the first row, bending on the second row, shearing on the third row, and their sum on the fourth row) [Colour figure can be viewed at wileyonlinelibrary.com] 
The energy content of bending elastic elements is less relevant than the other two forms of energy, so that in Figure 19H, we have that aforementioned cross deformation pattern typical of shear is reproduced for total energy. It has to be investigated, by means of a parametric analysis, if the pattern shown in Figure 19D can become dominant: we conjecture that this is the case and that, in this way, a suitable granular metamaterial could be eventually designed. This conjecture is confirmed by Figure 20 where the case $L=100$ is considered. This means that the boundary layer observed in Figure 19D starts from the bottom, invades the whole specimen when $\lambda$ increases, and there is no evidence of the cross type deformation pattern typical of shear. We remark also that in Figure 19, on the left column, which considers the case $\lambda=0.5$, the cross type pattern is perceptible, while on the contrary in Figure 20, on the left column, the layer-like pattern can be observed, especially in the bending energy part.

In Figure 21 (which report the case $L=10, \lambda=1$ for the absence, left column, and the presence, right column, of the hole), we can observe the growth of the boundary layer front, the hole as well as its interference with the boundary layers originating at the top and at the bottom of the specimen. We remark that:

1. For bending energy, the boundary layer originates mainly at the bottom of the specimen.

2. For extensional energy, the cross-like deformation pattern is clearly delineated and is slightly influenced by the presence of the hole;

3. For shear energy, one can observe that the deformation pattern in the case of absence of hole is changed by the presence of the hole, and that a perceptible boundary layer around the hole is generated; as $L=10$ is not big enough the classical cross-like pattern dominates for total energy; again, we expect that a careful parameters analysis may render dominant the layer-like pattern of Figure 21D also for such a value of $L$.

More complex, but also more interesting, is the analysis which is allowed by Figure $22(L=100$ and $\lambda=1)$. We present, simply as a preliminary series of considerations to be more deeply developed, the following remarks:

1. The comparison of Figure $22 \mathrm{G}$ and $22 \mathrm{H}$ shows that the presence of the holes relaxes the deformation pattern, by introducing a boundary counter-layer around the hole; however, the hole does not manage to establish any cross-like deformation pattern.

2. The highest effect on deformation patterns is observed in the shear energy plots (ie, Figure 22E and 22F) where the presence of holes determines a variation of about $50 \%$ of the average volume energy density; moreover, the counter-layer around the hole reaches the bottom and the top grains rows.

3. In Figure 22C and 22D (case of bending energy), the presence of the hole does not change the layer-like structure of deformation pattern; however, the boundary layer arising from the bottom is limited in thickness by the relaxation introduce by the hole; this also indicates the relevance, for non-Cauchy type of behaviour, of the bending interaction and energy.

4. In Figure 22A, one can distinguish a layer-like deformation pattern, while in Figure 22B, a cross-like deformation pattern is perceptible.

5. Around the hole, in the direction of shear displacement, one can distinguish a dipole of deformation energy; this indicates that if one wants to introduce a continuum model in which the material particle has the size of the considered hole, some generalisations of Cauchy scheme may be required; it is important to see that a relevant rotation of grains is present around the hole; it will be interesting in future investigations to establish how the presence of the holes change the rotation field of the grains.

Concerning Figure 21, we must remark that with $L=10$, the imposed shear displacement initiates, some boundary layers at the top, at the bottom of the assembly, and around the central imperfection. However, these boundary layers are not coalescing and remain concentrated around the corners and the central hole. When $L=100$, the boundary layers coalesce, and the deformation pattern and the deformation energy distribution assume a characteristic cross structure. There is a large difference between the deformation pattern in the case of presence of imperfection when compared with case of absence of imperfection even if plot of the resultant forces dependence on the imposed shear displacement becomes somehow different only when $L=100$.

\section{5 | Package with a large defect under linear displacement on the top}

As shown in Figure 23, which considers the package of grains with a large defect under imposed linear vertical displacements varying from $1.5 D_{g}$ (on the top-left) to $3 D_{g}$ (on the top-right) being $D_{g}$, the diameter of the grains, the reaction $R_{3}$ 


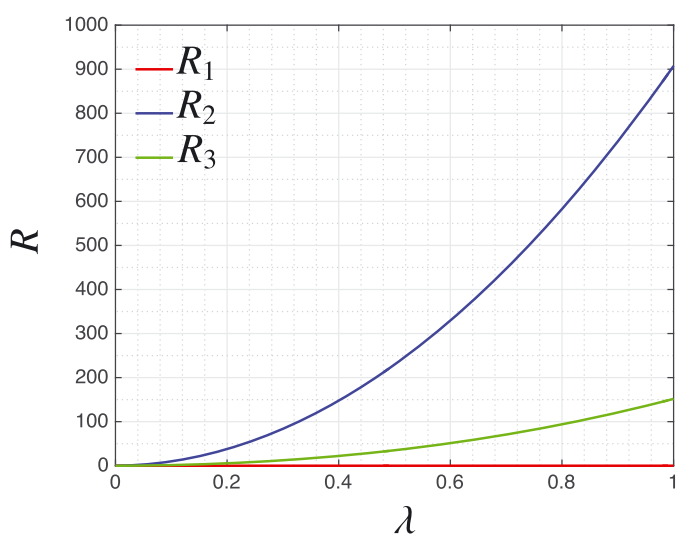

(A) $L=10$

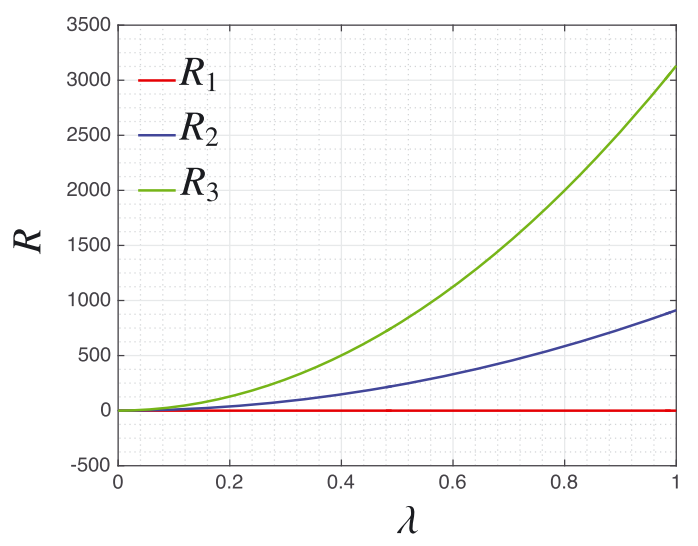

(B) $L=100$

FIGURE 23 Package of grains with one large defect under imposed vertical displacements varying from $1.5 D_{g}$ (on the top-left) to $3 D_{g}$ (on the top-right) being $D_{g}$ the diameter of the grains: reaction on the bottom side varying $\lambda$ for $\mathrm{A}, L=10$ and $\mathrm{B}, L=100$ [Colour figure can be viewed at wileyonlinelibrary.com]

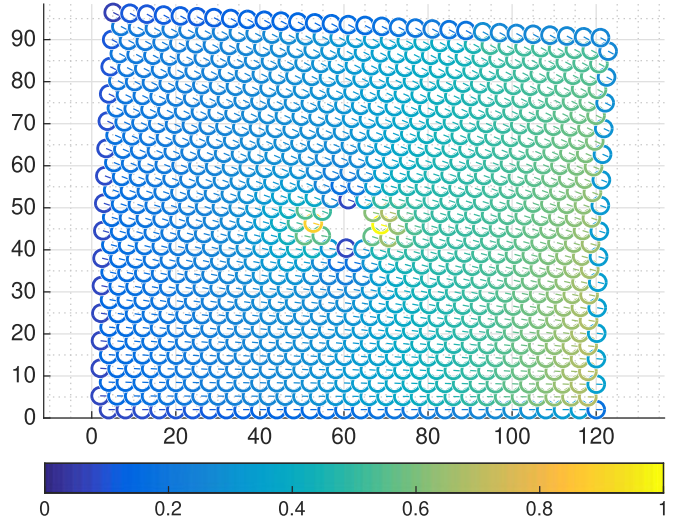

(A) $L=10$

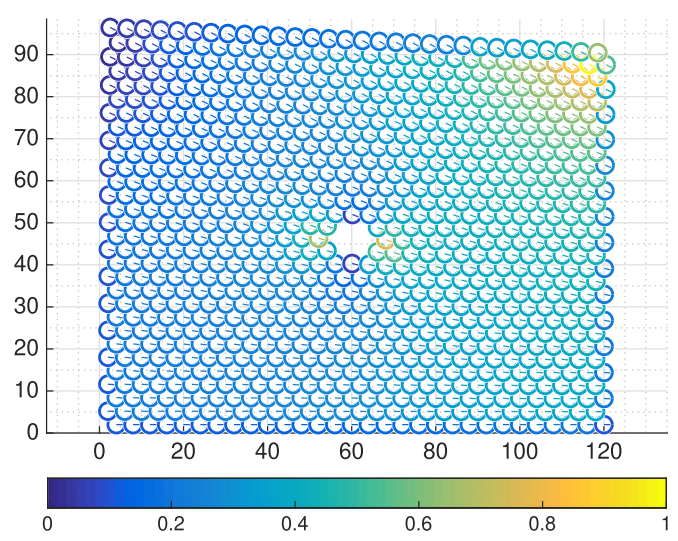

(B) $L=100$

FIGURE 24 Package of grains with one large defect under imposed vertical displacements varying from $1.5 D_{g}$ (on the top-left) to $3 D_{g}$ (on the top-right) being $D_{g}$ the diameter of the grains: deformations and strain energy (see the bar of colours) for $\lambda=1$ for A, $L=10$ and B, $L=100$ [Colour figure can be viewed at wileyonlinelibrary.com]

is comparable with the reactions $R_{1}$ and $R_{2}$ also in the case of compression and bending of the considered assembly of grains. When $L=10$, see Figure 23A, the vertical reaction $R_{2}$ is nearly 10 times bigger than $R_{3}$ (when $\lambda=1$ ), and one could possibly neglect Cosserat effects. However, when $L=100$, Figure 23B, this is not anymore possible as $R_{3}$ becomes more than three times bigger. The comparative analysis of the total deformation patterns shown in Figure 24A and 24B is very interesting. For the same imposed top grain row displacement:

1. When $L=10$, the highest deformation energy is concentrated in the lower right part of the specimen.

2. When $L=100$, the highest deformation energy is concentrated in the higher right higher part of the specimen.

A similar concentration of deformation energy is observed around the defect, with the typical dipole shape in both cases $L=10$ and $L=100$. The reader should remark that the scales of energy when $L=10$ and $L=100$ are different, so that our analysis is purely comparative for the deformation pattern. As Castigliano's theorem holds for all elastic linear and nonlinear systems, the values of reactive forces shown in Figure 23A and 23B indicate that the total value of deformation energy is higher from $L=100$, as expected.

We can conclude:

1. The considered REV, also when subject to compression and bending, as happened for shear, does not behave as a standard first gradient material point. 
2. If, on the considered REV boundary, grain rotations or grain relative displacements are imposed, see Section 4 , then the induced deformation, in the case of large characteristic lengths $L=100$, stores a large amount of energy, and the identification with a material point of a Cosserat or second gradient continuum seems needed, see Pavlov et al ${ }^{42}$ or dell'Isola et $\mathrm{al}^{43}$; moreover, the internal contact actions considered in generalised continua, see dell'Isola et al, ${ }^{35}$ seems necessary to describe observed phenomena.

\section{5 | CLOSING REMARKS AND FUTURE CHALLENGES}

The package of grains shown in Figure 4 can be regarded as a specific REV to be modeled as a material particle in a continuum model, if one is interested to phenomena whose characteristic length scale is much greater that its dimensions. On the other hand, one could try to introduce a continuum model, following a procedure like the one presented in dell'Isola et $\mathrm{al},{ }^{43}$ for describing the deformation of the considered assembly of grains: In this case, the continuum model is regarded as a description, which is alternative to the discrete one proposed for instance in this paper.

We remark that:

1. The distribution of deformation energy inside the considered assembly of grains may depend greatly on the presence of voids; the presence of a relatively large void (compared with the length scale) may change the form and intensity of boundary layers arising because of the presence of deformation energy related to rotational degree of freedom. For what concerns this modeling issue, we consider very interesting the comparison of our discrete model with the continuum generalised shell theories proposed, see previous studies..$^{4-46}$

2. It does not seem possible to obtain an effective continuum modeling without resorting to micro-structured continuum models, as those formulated in Germain. ${ }^{47}$

3. The presented results concerns uniquely the elastic deformation patterns; a careful comparison of the results, which can be obtained with the damage models based on discrete Lagrangian description, see Turco et al, ${ }^{48}$ and those obtained with the novel direct continuum modeling proposed in previous studies ${ }^{49-53}$ may be of interest.

4. The presented discrete micro-models can supply also a useful tool for helping in the determination of constitutive equations for continuum generalised macro-models, following the strategies presented in the previous studies. $^{14,15,54,55}$

In this paper, we have used a discrete model for a granular assembly with inter-granular interactions including shear, elongation, and bending. We have motivated with our numerical simulations qualitatively only the reasons for which a homogenised continuum model of our assembly of grains cannot be a first gradient continuum. Actually, we expect that in future developments of our investigations, the most suitable homogenised continuum model must be determined. In such a careful investigations, we will not only be obviously guided by the qualitative considerations presented here but also more complex theoretical considerations will be needed, see previous studies, ${ }^{34,37,56-59}$ in addition, see also dell'Isola et $\mathrm{al}^{60}$ for an historical framework and Reiher et $\mathrm{al}^{61}$ for a computational point of view.

On the other hand, the rigorous considerations will need to be supported also with heuristic considerations. In this aspect, the method of numerical homogenisation can be of use, see Giorgio ${ }^{54}$ and Khakalo et al. ${ }^{62}$ It has to be remarked that the numerical study of higher gradient continuum models is not so easy and requires some nontrivial improvements of the standard methods. We have found particularly interesting, in the context of the investigations presented here, the results found in the previous studies. ${ }^{63-65}$

We remark that we have not observed any locking phenomenon in the presented codes. It could be interesting to compare our physically based discretisation with that presented in Balobanov and Niiranen, ${ }^{66}$ which has the same interesting property. Remarkable are the results presented in Yaghoubi et al. ${ }^{67}$ We will accept the challenge to produce simulations based on Hencky-type discretisation, which can reproduce the dynamical effects which cited papers allows to treat in the dynamical case. The paper Khakalo and Niiranen ${ }^{68}$ is also of great relevance for our investigations: The reasons are obvious. We will carefully investigate the problem of comparing the perturbations induce by the presence of a defect when using our Hencky-type model and the discretisation of gradient model theory presented in the cited paper. In addition, we need to investigate how assemblies of nano-grains may resemble or be different from the assemblies, which we have considered here. In this context again, we have a ready algorithm and interesting results, ie, those presented in Khakalo and Niiranen. ${ }^{69}$ 
Finally, we list below some future challenges of this work:

1. Some technical applications require to considering inertia forces since the hypothesis of quasi-static application of external loads or given displacements is not close enough to describe their behaviour; in these cases, we can follow the guidelines reported in Giorgio et $\mathrm{al}^{70}$ and Engelbrecht and Berezovski. ${ }^{71}$

2. In this paper, we have chosen the stiffness parameters of the model with the only one goal to compare results deriving from different choices of the length scale; nevertheless, we are strongly interested to method capable to relate experiments or measures for identifying the stiffnesses of the proposed model, see, eg, Placidi et al. ${ }^{72}$

3. Here, we used the simplest law for the interaction of grains, however, other interaction laws may be used that are able to represent more complex behaviours of packages of grains, see, eg, previous studies. ${ }^{73-75}$

4. Since the considered package of grains can be regarded as a REV for continuum models, which surely have to be discretised, it appears interesting the interpolations base on B-splines and NURBS, see previous studies. ${ }^{76-80}$

5. Piola-Hencky models such as that presented in this work are the fundamental brick for building continuum models such as those presented, for example, in the previous studies ${ }^{43,81,82}$ or to extend this work towards three-dimensional packages of grains using the tools depicted in Turco $^{16}$ and Eugster et al ${ }^{83}$ or to study foams using the model presented in the previous studies ${ }^{84-86}$;

6. Finally, it could be interesting to consider peridynamics formulation as an alternative approach for the analysis of packages of grains, see, eg, Diyaroglu et $\mathrm{al}^{87}$ and De Meo et al..$^{88}$

\section{ORCID}

Emilio Turco (iD https://orcid.org/0000-0002-8263-7034

\section{REFERENCES}

1. Zhu HP, Zhou ZY, Yang RY, Yu AB. Discrete particle simulation of particulate systems theoretical developments. Chem Eng Sci. 2007;62:3378-3396.

2. Yohannes B, Gonzalez M, Abebe A, et al. Evolution of the microstructure during the process of consolidation and bonding in soft granular solids. Int J Pharm. 2016;503(1):68-77.

3. Li H, Lykotrafitis G. Two-component coarse-grained molecular-dynamics model for the human erythrocyte membrane. Biophys $J$. 2012;102:75-84.

4. Cundall PA, Strack ODL. A discrete numerical model for granular assemblies. 1979;29(1):47-65.

5. Kuhn MR. Granular Geomechanics. London-Kidlington: ISTE Press - Elsevier; 2017.

6. Radjaï F, Dubois F. Discrete-Element Modeling of Granular Materials. London: Wiley - ISTE; 2011.

7. Holtzman R, Silin DB, Patzek TW. Frictional granular mechanics. A variational approach. Int J Numer Methods Eng. 2010;81:1259-1280.

8. Misra A, Jiang H. Measured kinematic fields in the biaxial shear of granular media. Comput Geotech. 1997;20(3-4):267-285.

9. Richefeu V, Combe G, Viggiani G. An experimental assessment of displacement fluctuations in a 2D granular material subjected to shear. Geoté,chnique Lett. 2012;2:113-118.

10. Misra A, Poorsolhjouy P. Micro-macro scale instability in 2D regular granular assemblies. Continuum Mech Thermodyn. 2015;27(1-2):63-82.

11. Misra A, Placidi L, Turco E. Encyclopedia of Continuum Mechanics, Chapter Variational Methods for Continuum Models of Granular Materials. Berlin-Heidelberg: Springer; 2020.

12. Misra A, Poorsolhjouy P. Grain- and macro-scale kinematics for granular micromechanics based small deformation micromorphic continuum model. Mech Res Commun. 2017;81:1-6.

13. dell'Isola F, Maier G, Perego U, Andreaus U, Esposito R, Forest S. The Complete Works of Gabrio Piola: Volume I - Commented English Translation. Switzerland: Springer International Publishing; 2014.

14. Misra A, Poorsolhjouy P. Identification of higher-order elastic constants for grain assemblies based upon granular micromechanics. Math Mech Complex Syst. 2015;3(3):285-308.

15. Misra A, Poorsolhjouy P. Elastic behavior of 2D grain packing modeled as micromorphic media based on granular micromechanics. J Eng Mech. 2016;143(1):C4016005.

16. Turco E. Discrete is it enough? The revival of Piola-Hencky keynotes to analyze three-dimensional Elastica. Continuum Mech Thermodyn. 2018;30(5):1039-1057.

17. Turco E, dell'Isola F, Cazzani A, Rizzi NL. Hencky-type discrete model for pantographic structures: numerical comparison with second gradient continuum models. Zeitschrift fü,r Angewandte Mathematik und Physik. 2016;67(4):1-28.

18. Turco E, Caracciolo P. Elasto-plastic analysis of Kirchhoff plates by high simplicity finite elements. Comput Meth Appl Mech Eng. 2000;190:691-706. 
19. Hencky H. Über Die Angenäherte Lösung Von Stabilitätsproblemen Im Raum Mittels Der Elastischen Gelenkkette. PhD thesis: Engelmann; 1921.

20. Turco E. In-plane shear loading of granular membranes modeled as a Lagrangian assembly of rotating elastic particles. Mech Res Commun. 2018;92:61-66.

21. Darve F, Servant G, Laouafa F, Khoa HDV. Failure in geomaterials: continuous and discrete analyses. Comput Meth Appl Mech Eng. 2004;193(27-29):3057-3085.

22. Nicot F, Xiong H, Wautier A, Lerbet J, Darve F. Force chain collapse as grain column buckling in granular materials. Granular Matter. 2017;19(2):1-18.

23. Wautier A, Bonelli S, Nicot F. Micro-inertia origin of instabilities in granular materials. Int J Numer Anal Methods Geomech. 2018;42(9):1037-1056.

24. Riks E. An incremental approach to the solution of snapping and buckling problems. Int J Solids Struct. 1979;15:529-551.

25. Wriggers P. Nonlinear Finite Element Methods. Berlin-Heidelberg: Springer; 2008.

26. Turco E, Misra A, Pawlikowski M, dell'Isola F, Hild F. Enhanced Piola-Hencky discrete models for pantographic sheets with pivots without deformation energy: numerics and experiments. Int J Solids Struct. 2018;147:94-109.

27. Clarke MJ, Hancock GJ. A study of incremental-iterative strategies for non-linear analyses. Int J Numer Methods Eng. 1990;29:1365-1391.

28. Turco E, Barcz K, Pawlikowski M, Rizzi NL. Non-standard coupled extensional and bending bias tests for planar pantographic lattices. Part I: numerical simulations. Zeitschrift fü,r Angewandte Mathematik und Physik. 2016;67(122):1-16.

29. Turco E, Barcz K, Rizzi NL. Non-standard coupled extensional and bending bias tests for planar pantographic lattices. Part II: comparison with experimental evidence. Zeitschrift fü,r Angewandte Mathematik und Physik. 2016;67(123):1-16.

30. Turco E, Golaszewski M, Cazzani A, Rizzi NL. Large deformations induced in planar pantographic sheets by loads applied on fibers: experimental validation of a discrete Lagrangian model. Mech Res Commun. 2016;76:51-56.

31. Turco E, Rizzi NL. Pantographic structures presenting statistically distributed defects: numerical investigations of the effects on deformation fields. Mech Res Commun. 2016;77:65-69.

32. Turco E, Giorgio I, Misra A, dell'Isola F. King post truss as a motif for internal structure of (meta)material with controlled properties. Royal Soc Open Sci. 2017;4:171153.

33. Turco E, Golaszewski M, Giorgio I, D'Annibale F. Pantographic lattices with non-orthogonal fibres: experiments and their numerical simulations. Compos Part B: Eng. 2017;118:1-14.

34. Seppecher P. Moving contact lines in the Cahn-Hilliard theory. Int J Eng Sci. 1996;34(9):977-992.

35. dell'Isola F, Seppecher P, Madeo A. How contact interactions may depend on the shape of Cauchy cuts in Nth gradient continua: Approach “à, la D'Alembert”. Zeitschrift für angewandte Mathematik und Physik. 2012;63(6):1119-1141.

36. dell'Isola F, Seppecher P. The relationship between edge contact forces, double forces and interstitial working allowed by the principle of virtual power. Comptes Rendus de l'Academie de Sciences - Serie IIb: mecanique, Physique, Chimie, Astronomie. 1995;321:303-308.

37. Pideri C, Seppecher P. A second gradient material resulting from the homogenization of an heterogeneous linear elastic medium. Continuum Mech Thermodyn. 1997;9(5):241-257.

38. dell'Isola F, Goin H, Seppecher P. Radius and surface tension of microscopic bubbles by second gradient theory. Comptes Rendus de l'Academie de Sciences - Serie IIb: Mecanique, Physique, Chimie, Astronomie. 1995;320(5):211-216.

39. dell'Isola F, Forest S. Second gradient and generalized continua. A workshop held on 12-16 March 2012 in Cisterna di Latina. ZAMM - J Appl Math Mech / Zeitschrift fü,r Angewandte Mathematik und Mechanik. 2014;94(5):367-372.

40. Placidi L, Giorgio I, Della Corte A, Scerrato D. Euromech 563 Cisterna di Latina 17-21 March 2014 generalized continua and their applications to the design of composites and metamaterials: a review of presentations and discussions. Math Mech Solids. 2017;22(2):144-157.

41. Laudato M, Di Cosmo F. Euromech 579 Arpino 3-8 April 2017: generalized and microstructured continua: new ideas in modeling and/or applications to structures with (nearly) inextensible fibers-a review of presentations and discussions. Continuum Mech Thermodyn. 2018. https://doi.org/10.1007/s00161-018-0654-6:1--15

42. Pavlov IS, Potapov AI, Maugin GA. A 2D granular medium with rotating particles. Int J Solids Struct. 2006;43:6194-6207.

43. dell'Isola F, Giorgio I, Pawlikowski M, Rizzi NL. Large deformations of planar extensible beams and pantographic lattices: heuristic homogenisation, experimental and numerical examples of equilibrium. Proc R Soc London A Math Phys Eng Sci. 2016;472:1-23.

44. Altenbach H, Eremeyev VA. On the linear theory of micropolar plates. ZAMM - Journal of Applied Mathematics and Mechanics / Zeitschrift fu,r Angewandte Mathematik und Mechanik. 2009;89(4):242-256.

45. Eremeyev VA, Altenbach H. Shell-like Structures. Berlin-Heidelberg: Springer International Publishing; 2017.

46. Eremeyev VA, Pietraszkiewicz W. Material symmetry group and constitutive equations of micropolar anisotropic elastic solids. Math Mech Solids. 2016;21(2):210-221.

47. Germain P. The method of virtual power in continuum mechanics. Part 2: microstructure. SIAM J Appl Math. 1973;25(3):556-575.

48. Turco E, dell'Isola F, Rizzi NL, Grygoruk R, Müller WH, Liebold C. Fiber rupture in sheared planar pantographic sheets: numerical and experimental evidence. Mech Res Commun. 2016;76:86-90.

49. Placidi L, Barchiesi E. Energy approach to brittle fracture in strain-gradient modelling. Proc R Soc A Math Phys Eng Sci. 2018;474:1-19.

50. Giorgio I, Scerrato D. Multi-scale concrete model with rate-dependent internal friction. Eur J Environ Civil Eng. 2017;21(7-8):821-839.

51. Placidi L, Barchiesi E, Misra A. A strain gradient variational approach to damage: a comparison with damage gradient models and numerical results. Math Mech Complex Syst. 2018;6(2):77-100. 
52. Shirani M, Taheri Andani M, Kadkhodaei M, Elahinia M. Effect of loading history on phase transition and martensitic detwinning in shape memory alloys: limitations of current approaches and development of a 1d constitutive model. J Alloys Compd. 2017;729:390-406.

53. Ganghoffer JF, Pastrone F, eds.. Mechanics of Microstructured Solids: Cellular Materials, Fibre Reinforced Solids and Soft Tissues, Vol. 46: Berlin-Heidelberg: Springer Science Business Media; 2009.

54. Giorgio I. Numerical identification procedure between a micro Cauchy model and a macro second gradient model for planar pantographic structures. Zeitschrift fü,r Angewandte Mathematik und Mechanik. 2016;67(95):1-17.

55. Abali BE, Wu C-C, Müller WH. An energy-based method to determine material constants in nonlinear rheology with applications. Continuum Mech Thermodyn. 2016;28(5):1221-1246.

56. Pideri C, Seppecher P. A homogenization result for elastic material reinforced periodically with high rigidity elastic fibres. Comptes Rendus de l'Academie des Sci Ser IIB Mech Phys Chem Astron. 1997;8(324):475-481.

57. Scala R, Goethem NV. Constraint reaction and the Peach-Koehler force for dislocation networks. Math Mech Complex Syst. 2016;4(2).

58. Lurie SA, Kalamkarov AL. General theory of continuous media with conserved dislocations. Int J Solids Struct. 2007;44(22-23):7468-7485.

59. Gusev AA, Lurie SA. Symmetry conditions in strain gradient elasticity. Math Mech Solids. 2017;22(4):683-691.

60. dell'Isola F, Della Corte A, Giorgio I. Higher gradient continua: the legacy of piola, mindlin, sedov and toupin and some future research perspectives. Math Mech Solids. 2017;22(4):852-872.

61. Reiher JC, Giorgio I, Bertram A. Finite-element analysis of polyhedra under point and line forces in second-strain gradient elasticity. Journal of Engineering Mechanics. 2017;143(2):4016112-1-13.

62. Khakalo S, Balobanov V, Niiranen J. Modelling size-dependent bending, buckling and vibrations of $2 \mathrm{D}$ triangular lattices by strain gradient elasticity models: applications to sandwich beams and auxetics. J Eng Sci. 2018;127:33-52.

63. Niiranen J, Niemi AH. Variational formulations and general boundary conditions for sixth-order boundary value problems of gradient-elastic Kirchhoff plates. Eur J Mech - A/Solids. 2017;61:164-179.

64. Niiranen J, Balobanov V, Kiendl J, Hosseini SB. Variational formulations, model comparisons and numerical methods for Euler-Bernoulli micro- and nano-beam models; 2017;24(1):312-335.

65. Mousavi SM, Niiranen J, Niemi AH. Differential cubature method for gradient-elastic Kirchhoff plates. J Struct Mech. 2015;48(3):164-180.

66. Balobanov V, Niiranen J. Locking-free variational formulations and isogeometric analysis for the Timoshenko beam models of strain gradient and classical elasticity. Comput Methods Appl Mech Eng. 2018;339:137-159.

67. Yaghoubi ST, Balobanov V, Mousavi SM, Niiranen J. Variational formulations and isogeometric analysis for the dynamics of anisotropic gradient-elastic Euler-Bernoulli and shear-deformable beams. Eur J Mech - A/Solids. 2018;69:113-123.

68. Khakalo S, Niiranen J. Gradient-elastic stress analysis near cylindrical holes in a plane under bi-axial tension fields. Int J Solids Struct. 2017;110-111:351-366.

69. Khakalo S, Niiranen J. Form II of Mindlin's second strain gradient theory of elasticity with a simplification: for materials and structures from nano- to macro-scales. (to appear); 2018;71:292-319.

70. Giorgio I, Della Corte A, dell'Isola F. Dynamics of 1D nonlinear pantographic continua. Nonlinear Dyn. 2017;88(1):21-31.

71. Engelbrecht J, Berezovski A. Reflections on mathematical models of deformation waves in elastic microstructured solids. Math Mech Complex Syst. 2015;3(1):43-82.

72. Placidi L, Andreaus U, Della Corte A, Lekszycki T. Gedanken experiments for the determination of two-dimensional linear second gradient elasticity coefficients. Zeitschrift fü,r Angewandte Mathematik und Physik (ZAMP). 2015;66(6):3699-3725.

73. Braides A, Solci M. Asymptotic analysis of Lennard-Jones systems beyond the nearest-neighbour setting: a one-dimensional prototypical case. Math Mech Solids. 2016;21(8):915-930.

74. Atai A, Steigmann DJ. On the nonlinear mechanics of discrete networks. Archive Appl Mech. 1997;67(5):303-319.

75. Challamel N, Lerbet J, Wang CM. On buckling of granular columns with shear interaction: discrete versus nonlocal approaches. $J$ Appl Phys. 2014;115(23):234902.

76. Piegl L, Tiller W. The NURBS Book, 2nd ed. Springer-verlag: Berlin Heidelberg; 1997.

77. Cottrell JA, Hughes TJR, Bazilevs Y. Isogeometric Analysis: Toward Integration of CAD and FEA. Chichester: Wiley; 2009.

78. Greco L, Cuomo M, Contraffatto L, Gazzo S. An efficient blended mixed B-spline formulation for removing membrane locking in plane curved Kirchhoff rods. Comput Methods Appl Mech Eng. 2017;324:476-511.

79. Greco L, Cuomo M. B-spline interpolation of Kirchhoff-Love space rods. Comput Methods Appl Mech Eng. 2013;256:251-269.

80. Cuomo M, Contraffatto L, Greco L. A variational model based on isogeometric interpolation for the analysis of cracked bodies. Int J Eng Sci. 2014;80:173-188.

81. Giorgio I, Rizzi NL, Turco E. Continuum modelling of pantographic sheets for out-of-plane bifurcation and vibrational analysis. Proc $R$ Soc A Math Phys Eng Sci. 2017;473(20170636):1-21.

82. Shirani M, Luo C, Steigmann DJ. Cosserat elasticity of lattice shells with kinematically independent flexure and twist. Continuum Mech Thermodyn. 2018. https://doi.org/10.1007/s00161-018-0679-x

83. Eugster SR, Hesch C, Betsch P, Glocker C. Director-based beam finite elements relying on the geometrically exact beam theory formulated in skew coordinates. Int J Numer Methods Eng. 2014;97(2):111-129.

84. De Masi A, Merola I, Presutti E, Vignaud Y. Potts models in the continuum uniqueness and exponential decay in the restricted ensembles. J Stat Phys. 2008;133(2):281-345. 
85. De Masi A, Merola I, Presutti E, Vignaud Y. Coexistence of ordered and disordered phases in Potts models in the continuum. J Stat Phys. 2009;134(2):243-306.

86. Grimmett GR. Correlation inequalities for the Potts model. Math Mech Complex Syst. 2016;4(3-4):327-334.

87. Diyaroglu C, Oterkus E, Oterkus S, Madenci E. Peridynamics for bending of beams and plates with transverse shear deformation. Int $J$ Solids Struct. 2015;69:152-168.

88. De Meo D, Diyaroglu C, Zhu N, Oterkus E, Siddiq MA. Modelling of stress-corrosion cracking by using peridynamics. Int J Hydrogen Energy. 2016;41(15):6593-6609. 